\title{
Phased-Array Study of Dual-Flow Jet Noise: Effect of Nozzles and Mixers
}

Sang Soo Lee

QSS Group Inc., Cleveland, Ohio

James Bridges

Glenn Research Center, Cleveland, Ohio 


\section{NASA STI Program . . . in Profile}

Since its founding, NASA has been dedicated to the advancement of aeronautics and space science. The NASA Scientific and Technical Information (STI) program plays a key part in helping NASA maintain this important role.

The NASA STI Program operates under the auspices of the Agency Chief Information Officer. It collects, organizes, provides for archiving, and disseminates NASA's STI. The NASA STI program provides access to the NASA Aeronautics and Space Database and its public interface, the NASA Technical Reports Server, thus providing one of the largest collections of aeronautical and space science STI in the world. Results are published in both non-NASA channels and by NASA in the NASA STI Report Series, which includes the following report types:

- TECHNICAL PUBLICATION. Reports of completed research or a major significant phase of research that present the results of NASA programs and include extensive data or theoretical analysis. Includes compilations of significant scientific and technical data and information deemed to be of continuing reference value. NASA counterpart of peer-reviewed formal professional papers but has less stringent limitations on manuscript length and extent of graphic presentations.

- TECHNICAL MEMORANDUM. Scientific and technical findings that are preliminary or of specialized interest, e.g., quick release reports, working papers, and bibliographies that contain minimal annotation. Does not contain extensive analysis.

- CONTRACTOR REPORT. Scientific and technical findings by NASA-sponsored contractors and grantees.
- CONFERENCE PUBLICATION. Collected papers from scientific and technical conferences, symposia, seminars, or other meetings sponsored or cosponsored by NASA.

- SPECIAL PUBLICATION. Scientific, technical, or historical information from NASA programs, projects, and missions, often concerned with subjects having substantial public interest.

- TECHNICAL TRANSLATION. Englishlanguage translations of foreign scientific and technical material pertinent to NASA's mission.

Specialized services also include creating custom thesauri, building customized databases, organizing and publishing research results.

For more information about the NASA STI program, see the following:

- Access the NASA STI program home page at http://www.sti.nasa.gov

- E-mail your question via the Internet to help@sti.nasa.gov

- Fax your question to the NASA STI Help Desk at 301-621-0134

- Telephone the NASA STI Help Desk at 301-621-0390

- Write to:

NASA STI Help Desk

NASA Center for AeroSpace Information 7121 Standard Drive Hanover, MD 21076-1320 


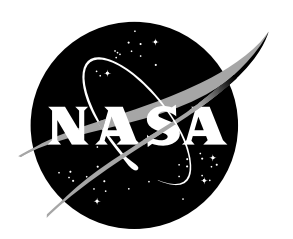

\section{Phased-Array Study of Dual-Flow Jet Noise: Effect of Nozzles and Mixers}

Sang Soo Lee

QSS Group Inc., Cleveland, Ohio

James Bridges

Glenn Research Center, Cleveland, Ohio

Prepared for the

12th AIAA/CEAS Aeroacoustics Conference

cosponsored by the American Institute of Aeronautics and Astronautics and Confederation of European Aerospace Societies

Cambridge, Massachusetts, May 8-10, 2006

National Aeronautics and

Space Administration

Glenn Research Center

Cleveland, Ohio 44135 
Level of Review: This material has been technically reviewed by technical management.

Available from

NASA Center for Aerospace Information

7121 Standard Drive

Hanover, MD 21076-1320
National Technical Information Service 5285 Port Royal Road Springfield, VA 22161

Available electronically at http://gltrs.grc.nasa.gov 


\title{
Phased-Array Study of Dual-Flow Jet Noise: Effect of Nozzles and Mixers
}

\author{
Sang Soo Lee \\ QSS Group, Inc. \\ Cleveland, Ohio 44135 \\ James Bridges \\ National Aeronautics and Space Administration \\ Glenn Research Center \\ Cleveland, Ohio 44135
}

\begin{abstract}
A 16-microphone linear phased-array installed parallel to the jet axis and a 32-microphone azimuthal phasedarray installed in the nozzle exit plane have been applied to identify the noise source distributions of nozzle exhaust systems with various internal mixers (lobed and axisymmetric) and nozzles (three different lengths). Measurements of velocity were also obtained using cross-stream stereo particle image velocimetry (PIV). Among the three nozzle lengths tested, the medium length nozzle was the quietest for all mixers at high frequency on the highest speed flow condition. Large differences in source strength distributions between nozzles and mixers occurred at or near the nozzle exit for this flow condition. The beamforming analyses from the azimuthal array for the 12-lobed mixer on the highest flow condition showed that the core flow and the lobe area were strong noise sources for the long and short nozzles. The 12 noisy spots associated with the lobe locations of the 12-lobed mixer with the long nozzle were very well detected for the frequencies $5 \mathrm{KHz}$ and higher. Meanwhile, maps of the source strength of the axisymmetric splitter show that the outer shear layer was the most important noise source at most flow conditions. In general, there was a good correlation between the high turbulence regions from the PIV tests and the high noise source regions from the phased-array measurements.
\end{abstract}

\section{Introduction}

This report presents the results of the phased-array test cosponsored by the NASA Quiet Aircraft Technology program and Rolls Royce Corporation. This test was conducted in the Nozzle Acoustic Test Rig (NATR) in the NASA Glenn Research Center by using the model hardware first tested in 1996 (Mengle, Baker and Dalton, 2002). This model hardware represented a turbofan engine exhaust configuration employing forced mixing between the secondary fan and primary core flow streams. Three nozzles and six mixer configurations including those producing the lowest and highest measured noise levels from the previous test were tested. These configurations were tested on subsonic operating conditions.

The far field noise data obtained during the previous 1996 tests showed a wide variation in the far field noise levels for a fixed thrust level. While certain general trends could be identified from this data, it was not possible to explain these trends in detail. Therefore, in this test, additional diagnostic measurements were used to help improve our understanding of the noise generation process when forced mixing was present. The results from the phasedarray analyses are presented in this paper and in Lee (2005). Meanwhile, results from the PIV tests were presented in Bridges and Wernet (2004) and the analysis of the far-field acoustics were given by Tester, Fisher, and Dalton (2004), Garrison, Dalton, Lyrintzis, and Blaisdell (2004) and Tester and Fisher (2005). The CFD results on the same mixers and nozzles were presented in Garrison, Lyrintzis, Blaisdell, and Dalton (2005).

\section{Experimental Setup}

\subsection{Test Hardware and Test Conditions}

The test was conducted in the NASA Glenn Research Center AeroAcoustic Propulsion Laboratory (AAPL). The AAPL was a $65 \mathrm{ft}$ radius anechoic hemispherical dome. Its walls and floor area were covered by acoustic wedges. The Nozzle Aeroacoustic Test Rig (NATR) in the AAPL provides the airflow for the flight simulation capability. The NATR duct was acoustically lined and consists of an annular ejector system connected to a plenum followed by the transition section which was an ASME long-radius, low beta venturi nozzle. This flow was exhausted through a second nozzle of 53 in. diameter which forms a free jet to simulate the effects of forward flight on the test article. The centerline of the free jet was $10 \mathrm{ft}$ above the floor. An acoustically treated wall separates the NATR from the section of the building which does not have acoustic treatment on the floor. 
Downstream of the NATR was the Dual Flow Jet Exit Rig (DFJER2). The DFJER2 was the structure through which airflow was delivered from the facility compressed air system to the test article. This hardware has been recently redesigned and refitted in order to reduce the levels of internal flow noise. The test article was attached to the aft end of the DFJER2.

The mixed flow exhaust system model hardware consists of co-annular fan and core flow ducts, forced mixer and exhaust nozzle. Three different nozzles, L0, L1, and L2 were tested. The nozzle lengths from the mixer plane were $1.53,1.16$, and 0.78 nozzle diameters, respectively, as shown in figure 2.1. All nozzles have a nominal cold throat diameter of 7.245 in. and a nominal cold throat area of 41.225 sq. in.

Figure 2.2 shows the pictures of the Confluent axisymmetric splitter and five configurations of lobed mixer tested in this study. These configurations represent a substantial variation of the important geometric parameters of a lobed mixer. The Confluent axisymmetric splitter, Con, served as the baseline configuration. Three 12-lobed mixers were tested in order to understand the effect of mixer penetration. The low, intermediate, and high penetration mixers, $12 \mathrm{CL}, 12 \mathrm{UM}$, and $12 \mathrm{UH}$ have peak to peak penetrations of $0.5,0.7$, and 0.88 of the mixer plane annulus, respectively. Two 20-lobed mixers, 20UH and 20DS were used to study the effect of lobe width and scallop. Both 20-lobed mixers have the same high penetration as the 12UH mixer. The 20DS mixer has deep scallops.

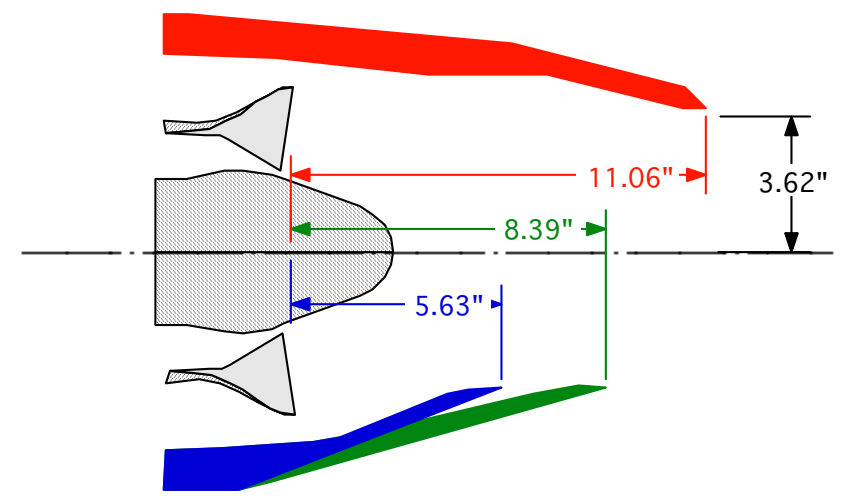

Figure 2.1.-Dimensions and internal contours of nozzles L0 (red), L1 (green), and L2 (blue).

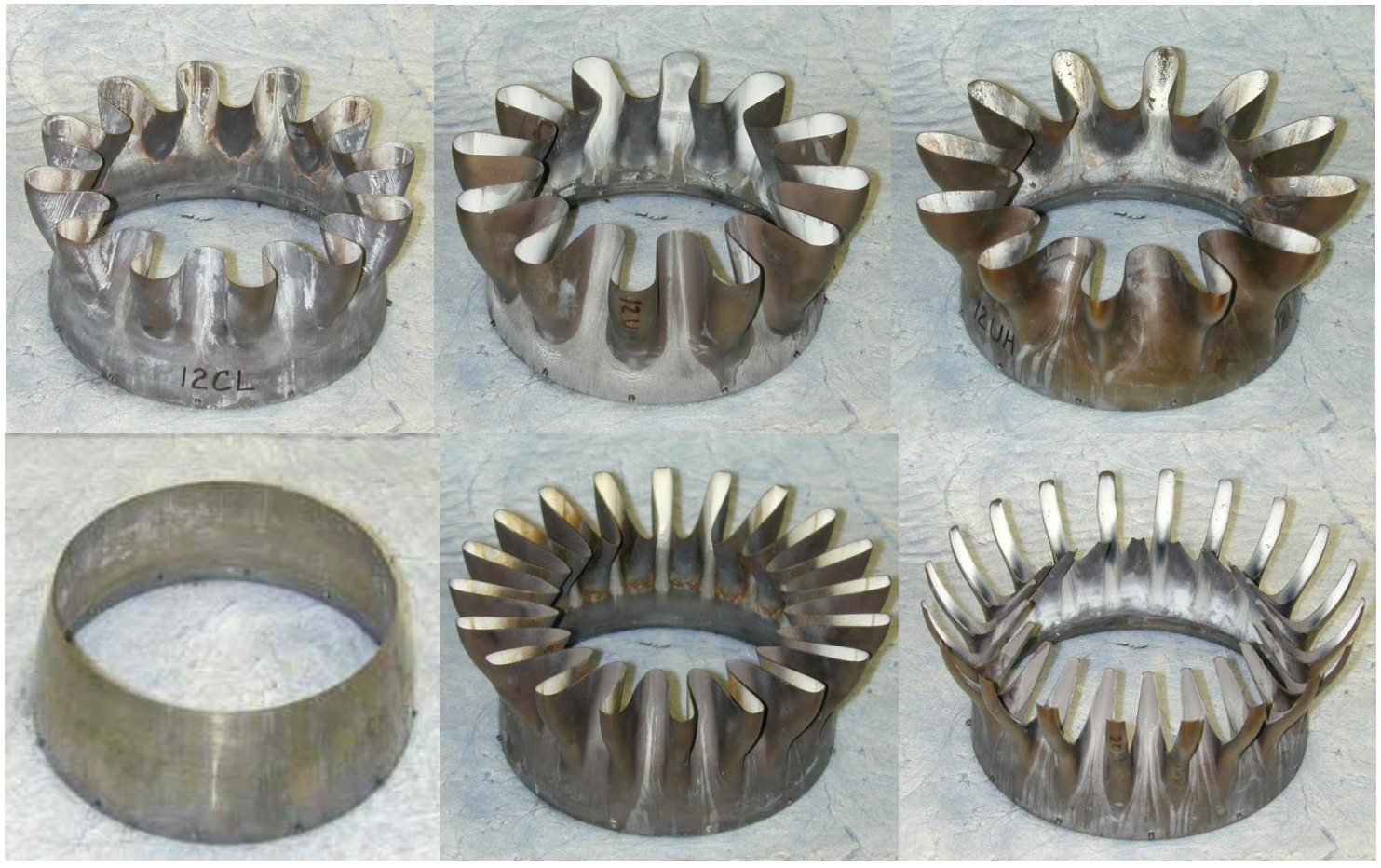

Figure 2.2.-Pictures of mixers: 12CL (top left), 12UM (top center), 12UH (top right), Con (bottom left), 20UH (bottom center), and 20DS (bottom right). 
Phased-array beamforming results from three flow conditions are presented in this paper. The core and fan (bypass) flow conditions of the set points 110 and 310 are listed in table 2.1. Set point 5000, where both core and fan streams have the same pressure ratio without heating, was used to mimic a single flow cold jet for comparison purposes. There was no free jet in these three set points. The fully mixed conditions for these flows were given in Tester, Fisher, and Dalton (2004) and Tester and Fisher (2005).

TABLE 2.1.-DEFINITION OF SET POINTS AND FLOW CONDITIONS

\begin{tabular}{|c|c|c|c|c|c|}
\hline Set point id & Core NPR & Fan NPR & Core NTR & Fan NTR & Free jet Mach No \\
\hline 110 & 1.39 & 1.44 & 2.799 & 1.196 & 0 \\
\hline 310 & 1.74 & 1.82 & 3.337 & 1.196 & 0 \\
\hline 5000 & 1.435 & 1.435 & 1 & 1 & 0 \\
\hline
\end{tabular}

Overall rig operational parameters were monitored and recorded through the facility ESCORT data system. Each data point was identified by the Escort number. The actual flow conditions along with the atmospheric pressure, temperature and humidity were given in Lee (2005). More detailed information on the test hardware and flow conditions can also be found in Bridges and Wernet (2004), Tester, Fisher, and Dalton (2004) and Tester and Fisher (2005).

\subsection{Sixteen-Microphone Linear Phased Array}

The linear phased array was composed of 16 microphones positioned at unequal intervals. Two different locations of the phased-array system, one mounted on the floor and the other mounted in the overhead position as shown in figure 2.3. Both arrays were installed roughly parallel to the jet-flow centerline. The microphones in the floor array were flush mounted without the protective grids, while those in the overhead array were mounted on microphone holders and the protective grids were used.

Microphone positions in both array systems are shown in figure 2.4 for the L0 nozzle. The origin of the righthanded Cartesian coordinate system was located at the center of the nozzle exit plane. The $\mathrm{x}$ coordinate was on the jet centerline with positive values in the jet-flow downstream direction. The $\mathrm{x}$ and $\mathrm{y}$ coordinates were parallel with and the $\mathrm{z}$ coordinate was perpendicular to the floor. The microphone number 1 of the floor array was located in the upstream location, but the numbering of microphones was reversed for the overhead array system. The z-coordinates of the floor microphones were about $-15 \mathrm{D}$, where $\mathrm{D}$ represents the nozzle diameter of $7.245 \mathrm{in}$., and the distance between the floor microphone 1 and the L0 nozzle center was about 18D. The z-coordinates of the overhead microphones were about $14.5 \mathrm{D}$ and the distance between the overhead microphone 16 and the L0 nozzle center was about 18D. The exact coordinates of the microphones are available in Lee (2005).

Since the lengths of the nozzles were different, the axial microphones positions in figure 2.4 should be increased by $0.37 \mathrm{D}$ and $0.75 \mathrm{D}$ for the L1 and L2 nozzles, respectively.

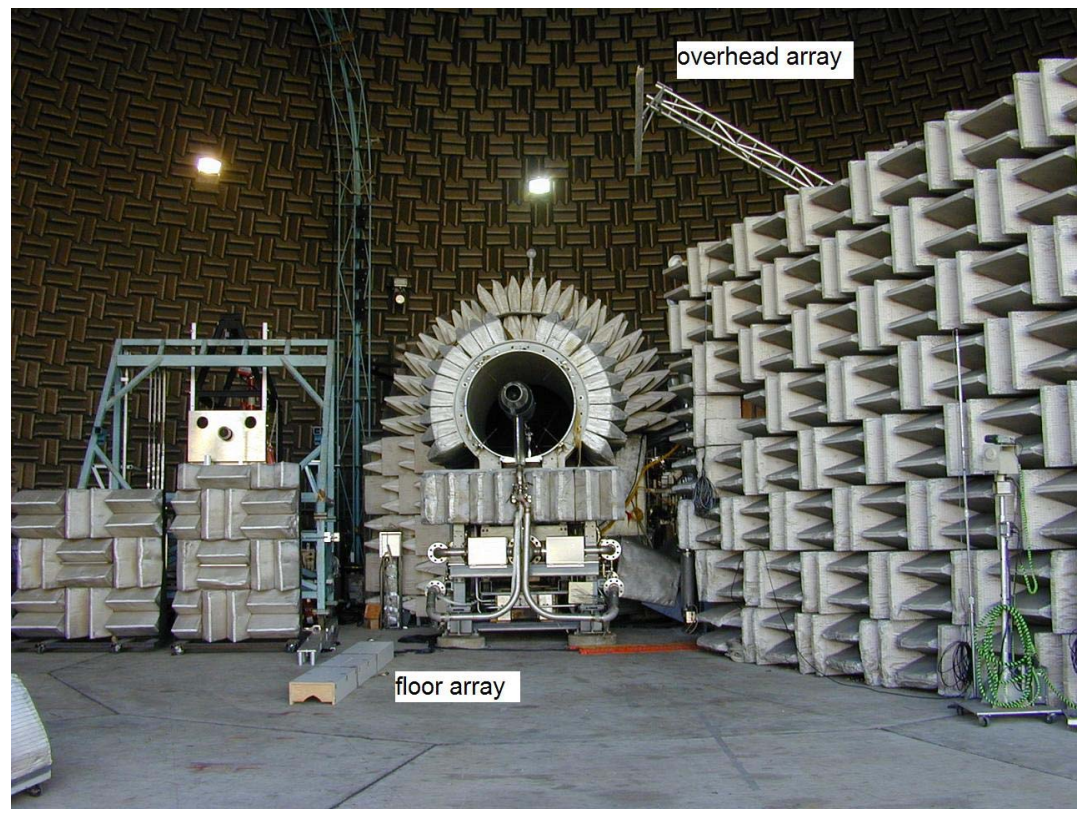

Figure 2.3.-Floor and overhead linear phased-array systems. 

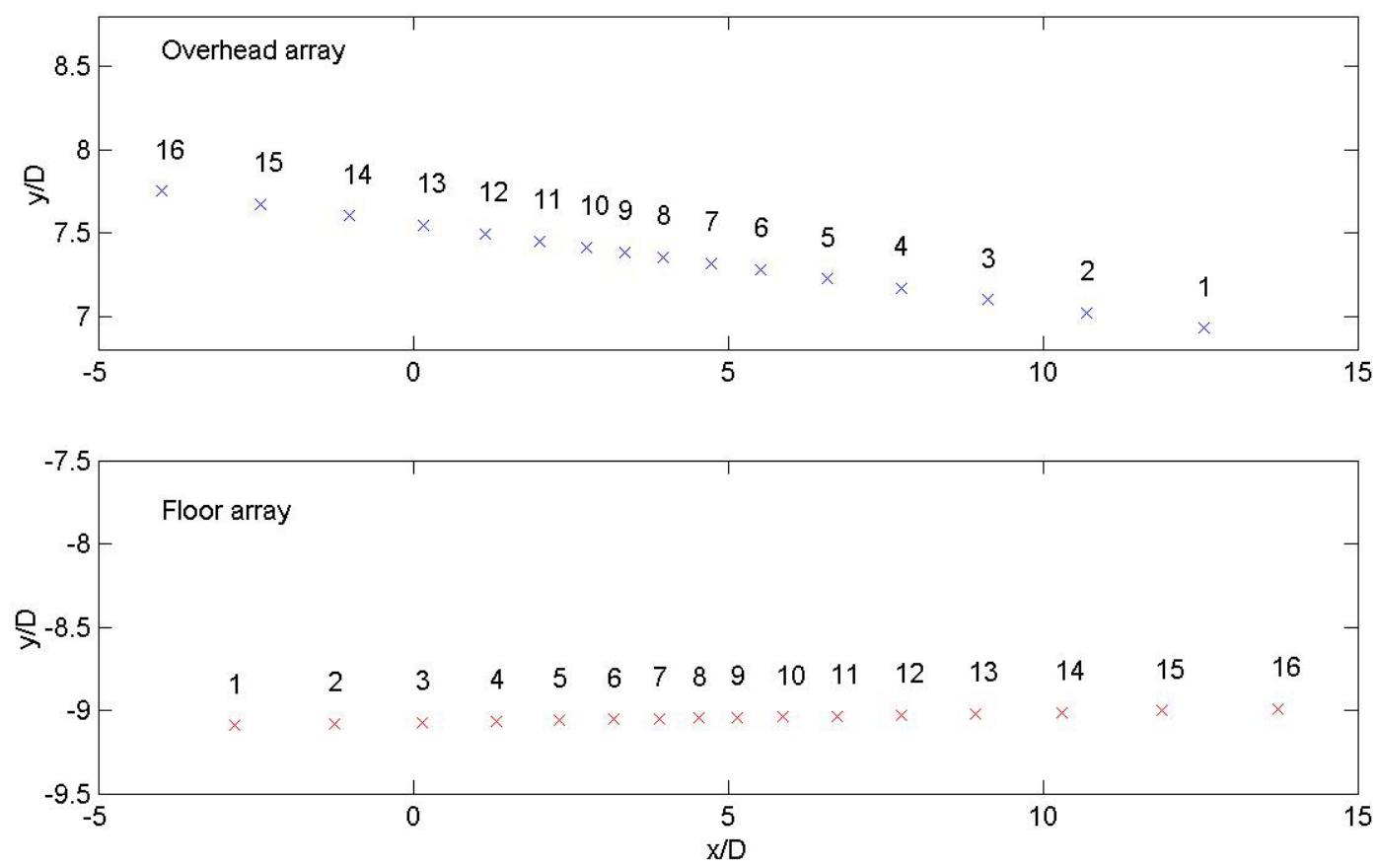

Figure 2.4.- Microphone positions in floor and overhead linear arrays for the L0 nozzle.

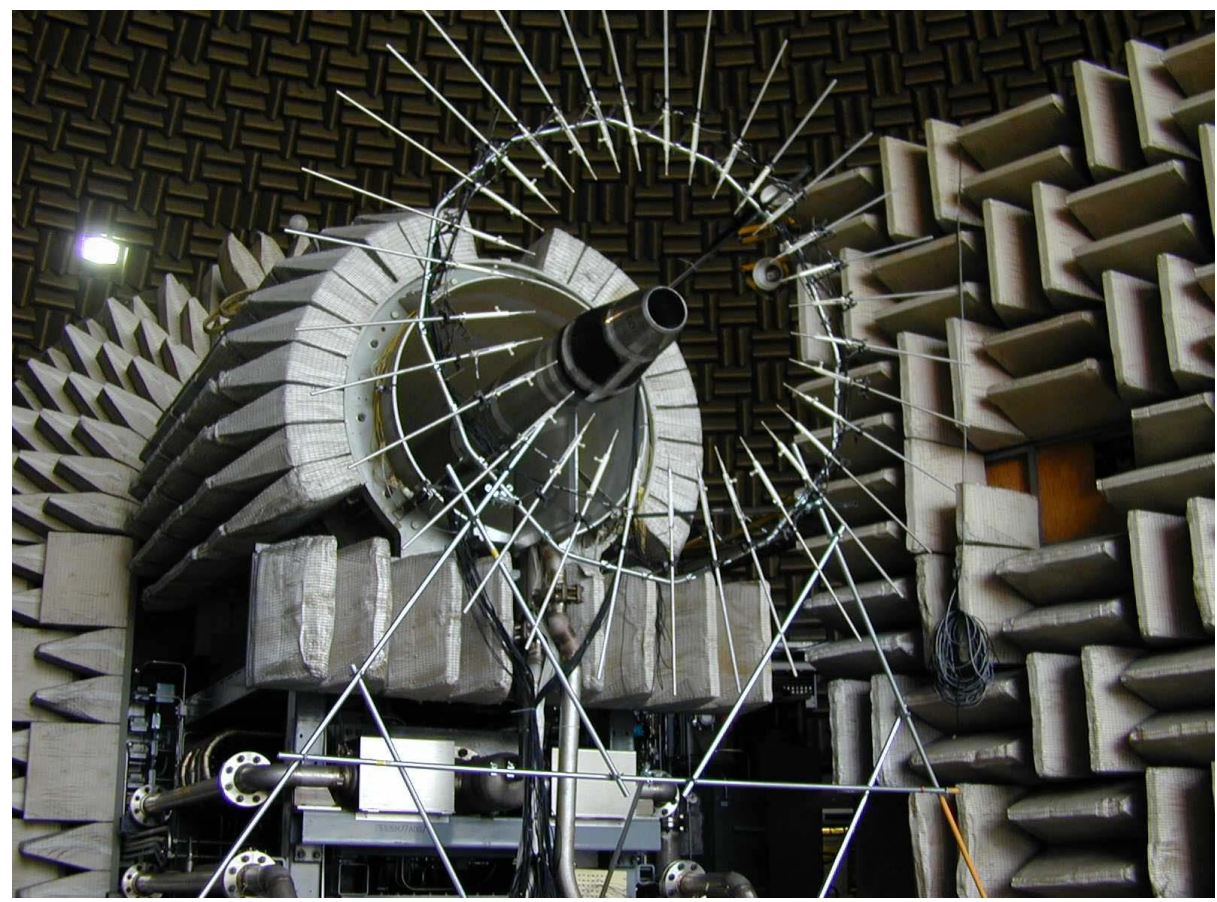

Figure 2.5.-Azimuthal phased-array system.

\subsection{Thirty Two Microphone Azimuthal Phased Array}

In an attempt to resolve the noise source distribution in the cross section of a jet, an azimuthal phased-array system has been developed. As shown in figures 2.5 and 2.6, 32 microphones were installed on the 6.63D (4 ft) diameter circle at $11.25^{\circ}$ intervals. The streamwise position of the microphone plane was fixed at the L0 nozzle exit. Therefore, the x-coordinates of the microphone positions become $0,0.37 \mathrm{D}$ and $0.75 \mathrm{D}$ for the L0, L1, and L2 nozzles, respectively. Protective grids were used on these microphones. 


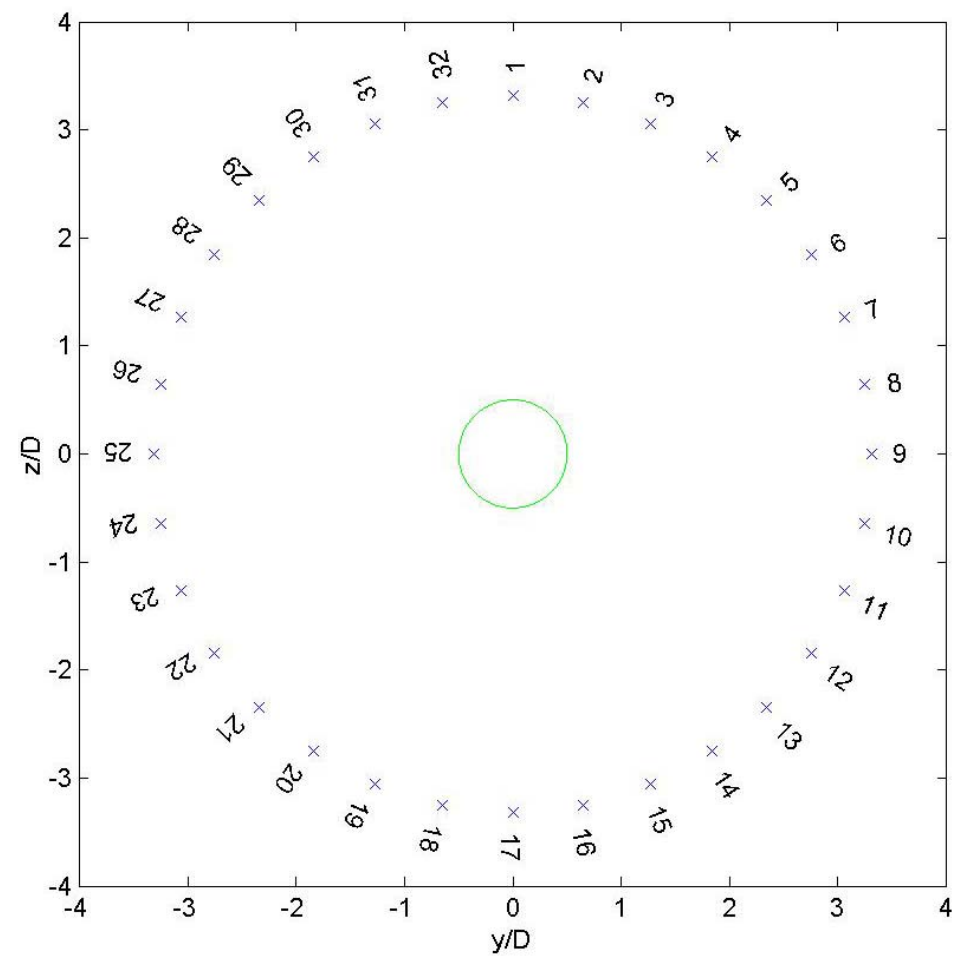

Figure 2.6.-Microphone positions of the azimuthal phased-array system. The green circle represents the nozzle exit.

\subsection{Data Processing}

Free-field 1/4 in. condenser microphones were used to measure sound pressure level. The microphone preamplifiers were connected to the four-channel microphone conditioning amplifiers, B\&K Nexus 2690 units. The outputs of the Nexus 2690 were connected into the Datamax data acquisition system. The sampling rate of the Datamax system was $200 \mathrm{KHz}$ with the analog bandwidth of $90 \mathrm{KHz}$ for the 16-microphone linear arrays and was $100 \mathrm{KHz}$ with the analog bandwidth of $40 \mathrm{KHz}$ for the 32-microphone azimuthal array. All microphone signals were recorded simultaneously for $40 \mathrm{sec}$. The calibration constants of microphones were obtained by using a $250 \mathrm{~Hz}$ single frequency piston phone. The atmospheric attenuations were not compensated in the results since they were not very important within the frequency ranges considered in this paper. The 'as measured' signals were used to compute narrowband spectra and beamforming outputs. For the cross-spectral density calculations, the Matlab function 'csd' was used with 8192 data points, 50 percent overlapping and Hanning's periodic window. All beamforming analyses have been performed based on the narrowband cross-spectral densities with bandwidth of $24.41 \mathrm{~Hz}$ or $12.21 \mathrm{~Hz}$ for the linear and azimuthal arrays, respectively.

The classical beamforming method described in Lee (2005), the same as in Dougherty (2002), Underbrink (2002) and Johnson and Dudgeon (1993), was used for the beamforming level calculations.

\section{Point Sound Source Response}

\subsection{Point Noise Source Response of the Linear Arrays}

A point source was used to register and confirm the array dimensions and processing. The beamforming results from the floor array with an impinging jet located at about one nozzle diameter from the L0 nozzle exit are given in figure 3.1. Compressed air from a 1/4 in. nozzle was impinged on a small perpendicular plate to mimic a point sound source. The classical beamforming method was used to calculate the beamforming levels along the jet centerline for all narrowband frequencies. At each frequency the peak location and peak level, given in the first two columns in the second row, were found from the streamwise location where the maximum beamforming level occurs. The contour plots of beamforming levels are given in the first row in figure 3.1. The beamforming levels, normalized by the peak level at each frequency, are used in the upper-left contour plot with $10 \mathrm{~dB}$ color bar scale. Meanwhile, the contour plot of the absolute beamforming levels is shown in the upper-right figure with the color bar in $\mathrm{dB}$ scale. The 

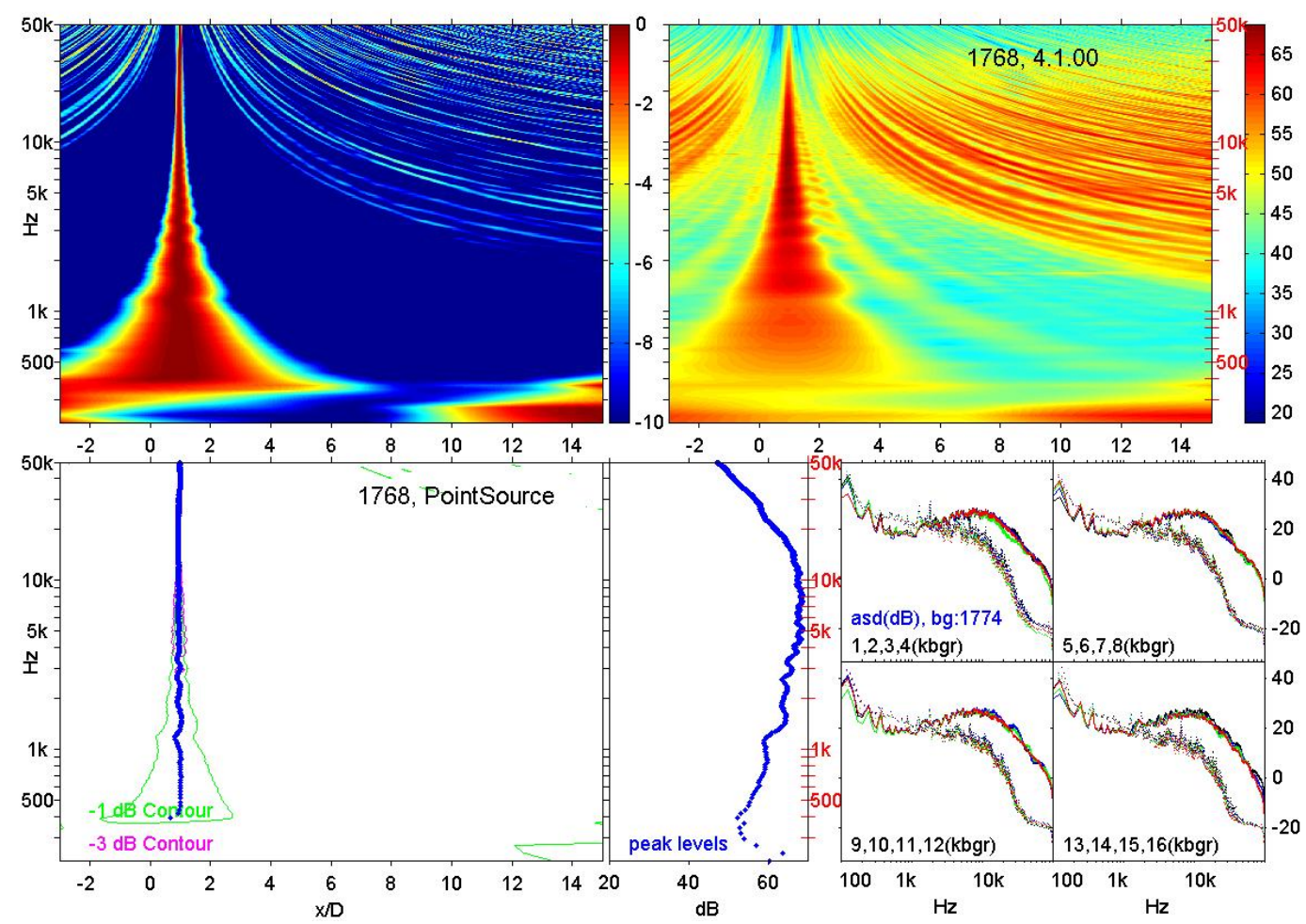

Figure 3.1.-Classical beamforming results of impinging jet noise source with floor array (Escort number: 1768).

narrowband spectra for all 16 microphones are plotted in the bottom-right figures. Those of the back ground noise (Escort number, 1774) are plotted with dotted curves.

The floor array was very successful at finding noise source location, as seen in the figure. Since the microphone positions were accurately known, the beamforming analysis can produce accurate peak locations up to the Nyquist critical frequency of $100 \mathrm{KHz}$. The noise level produced by the impinging jet drops below the background level for frequencies below $1 \mathrm{KHz}$ as shown in the narrowband spectra.

Figure 3.2 shows the beamforming results when an acoustic sound source was used for the same floor array. One end of a $10 \mathrm{ft}$ long steel pipe of one inch diameter was connected to an acoustic driver and the other open end was positioned at about 1.2D from the nozzle exit on the jet centerline. The acoustic driver was driven by an amplifier and white noise generator. In the figure it is shown that, above $400 \mathrm{~Hz}$, the peak source locations were accurately found. Even though the acoustic driver does not produce any meaningful sound beyond $20 \mathrm{KHz}$, compared to the background noise, the position of the sound-pipe opening was very well located up to $50 \mathrm{KHz}$. Between the impinging jet results and that of the acoustic source, the floor array was demonstrated to accurately assess the noise source location for frequencies as high as $50 \mathrm{KHz}$.

The classical beamforming results of the overhead array for the acoustic sound source are given in figure 3.3. The location of the acoustic sound source was successfully found in the frequency range between $300 \mathrm{~Hz}$ and $40 \mathrm{KHz}$.

The array patterns of both floor and azimuthal arrays can be found in Lee (2005). 


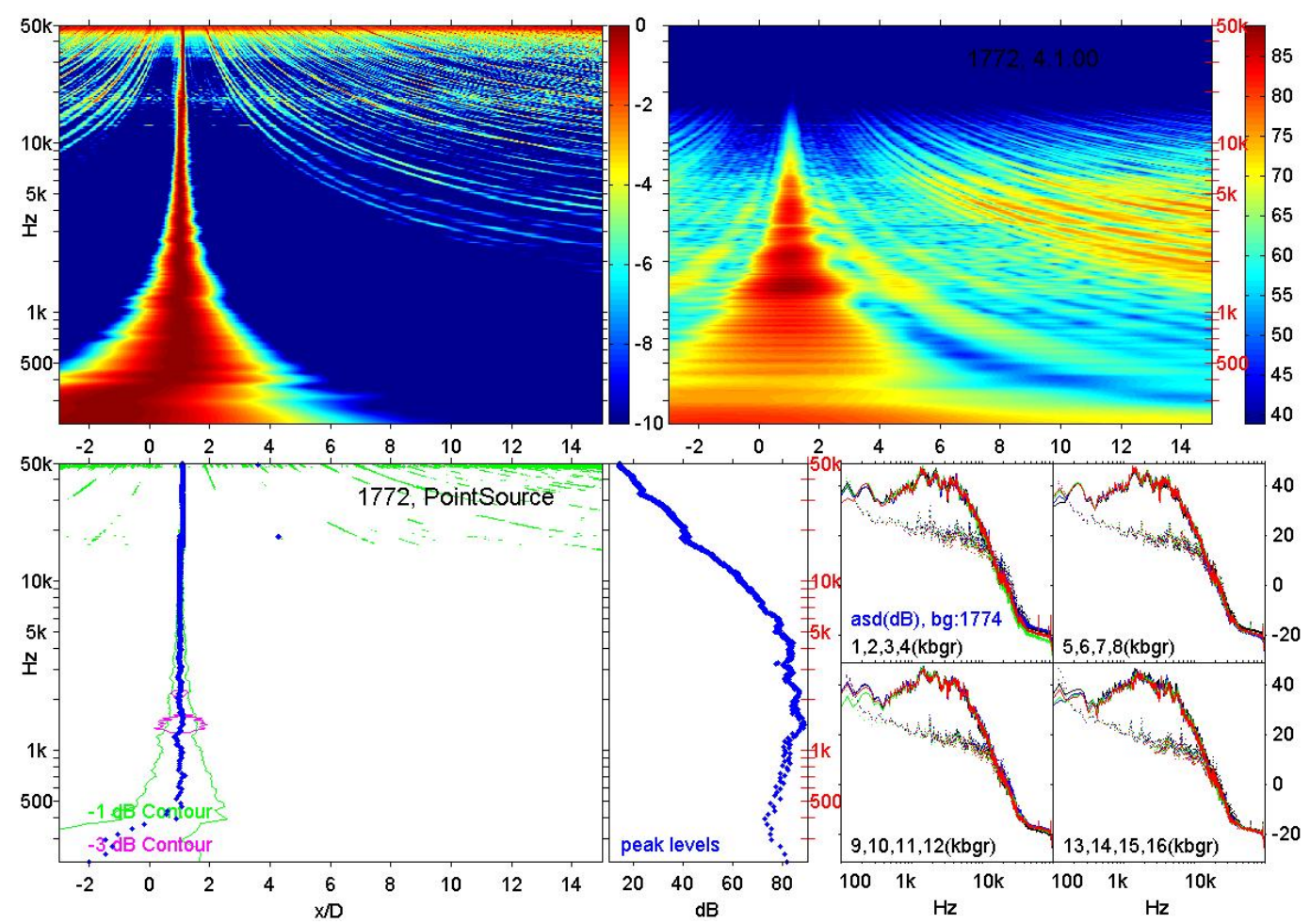

Figure 3.2.-Classical beamforming results of white noise acoustic sound source with floor linear array (Escort number: 1772).

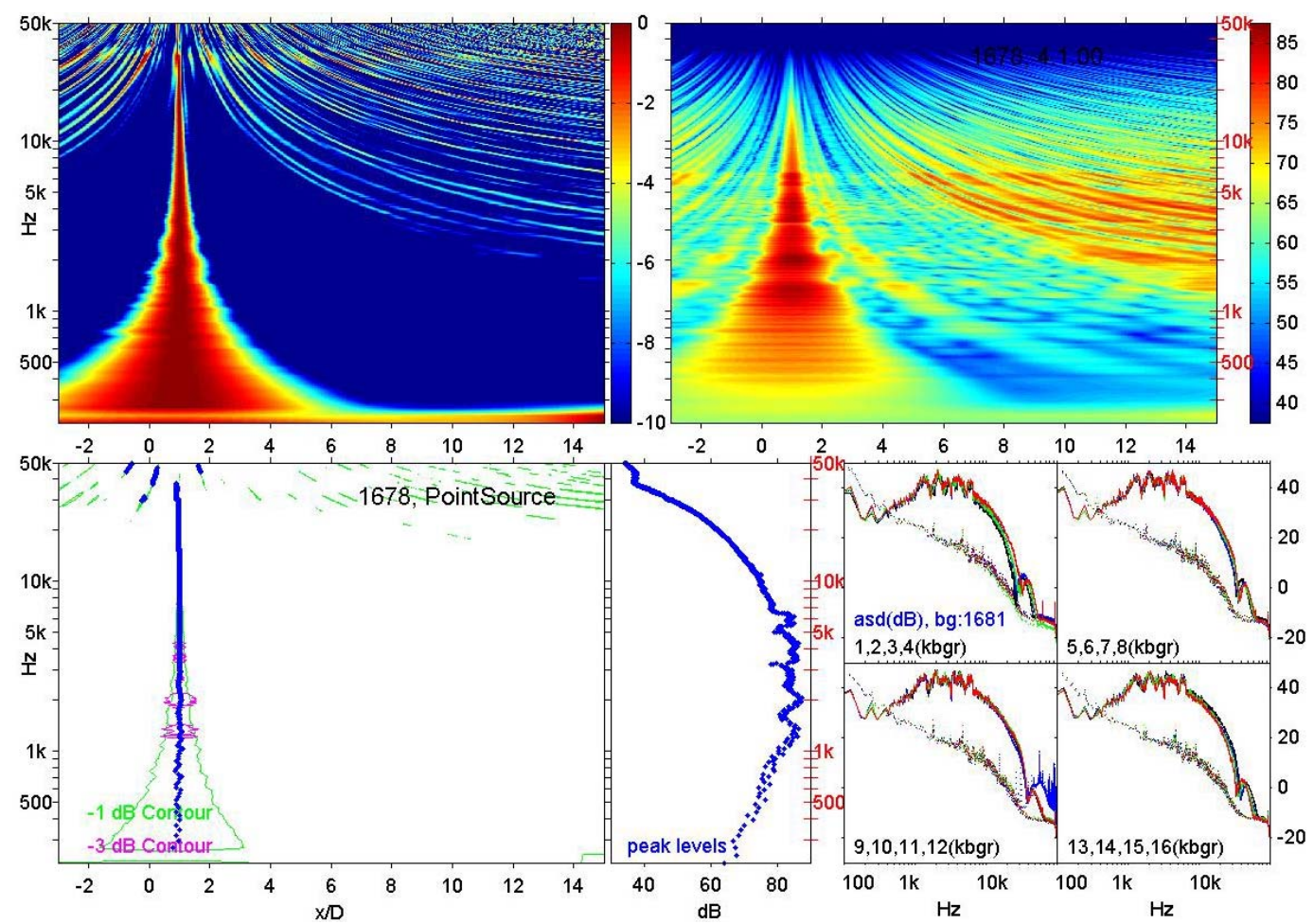

Figure 3.3.-Classical beamforming results of white noise acoustic sound source with overhead linear array (Escort number: 1678). 

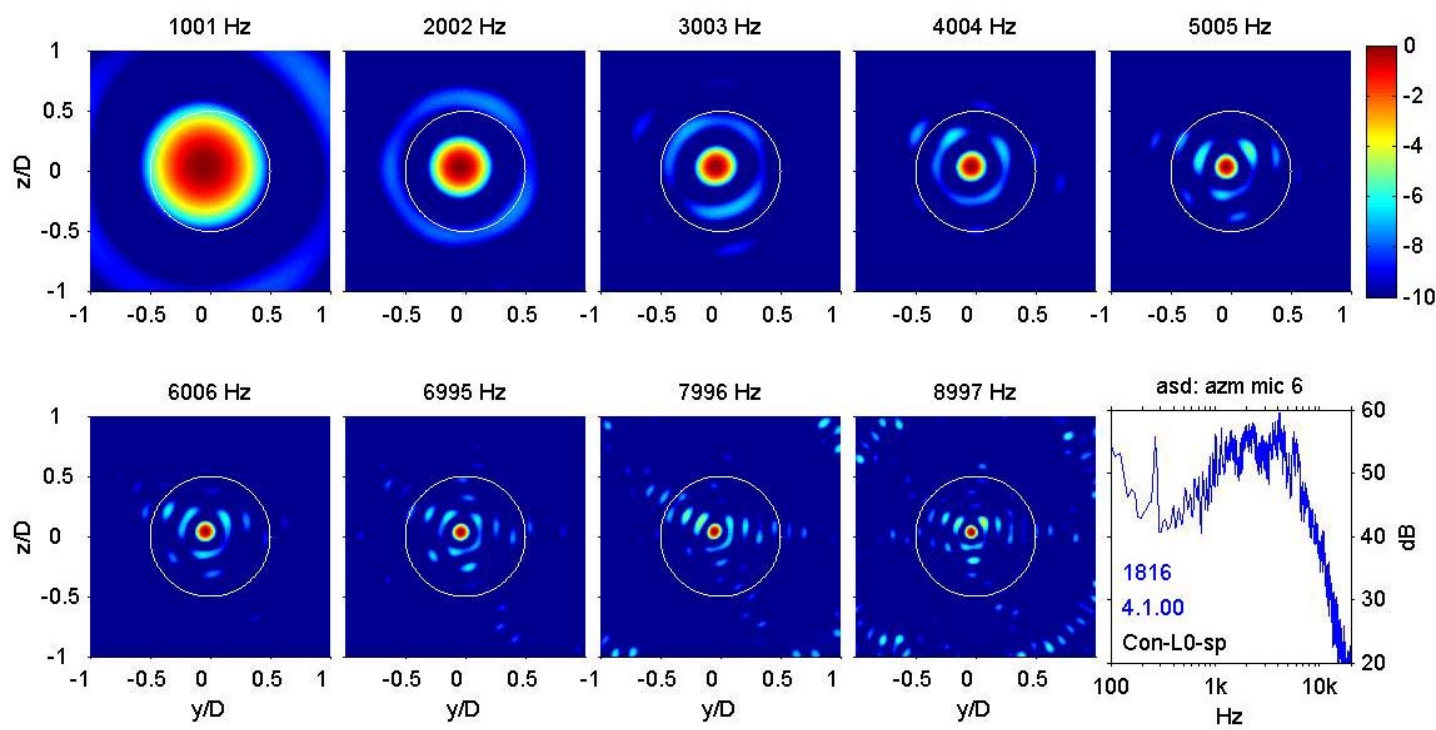

Figure 3.4.- Classical beamforming results of white noise acoustic sound source with azimuthal array (Escort number: 1816).

\subsection{Point Sound Source Response of the Azimuthal Array}

Figure 3.4 shows the classical beamforming results from the azimuthal array with the acoustic sound source. The opening of the sound-pipe was located at $\mathrm{x}=0.4 \mathrm{D}$ from the $\mathrm{L} 0$ nozzle exit, pointing toward the nozzle, and near the center of the nozzle exit plane. The beamforming levels at nine narrowband frequencies were computed in the $y-$ $\mathrm{z}$ cross-section at $\mathrm{x}=0.4 \mathrm{D}$. The contour plots of the normalized beamforming levels, by the peak value at each frequency, are given in figure 3.4 with the $10 \mathrm{~dB}$ color bar scale. The sound source location was accurately predicted at all frequencies.

\section{Classical Beamforming Results From the Linear Arrays \\ 4.1 Effect of Nozzle on 12CL Mixer}

The results of the linear phased array analyses are presented for the 12CL mixer that has the lowest penetration among three 12-lobed mixers tested in this study. Figure 4.1 shows the narrowband spectra with the bandwidth of $24.41 \mathrm{~Hz}$ from the microphone numbers $13,10,5$, and 1 of the overhead array. The corresponding microphone angles from the center of the $\mathrm{L} 0$ nozzle exit plane were $91^{\circ}, 100^{\circ}, 112^{\circ}$, and $128^{\circ}$, respectively. Since the lengths of nozzles were different and the overhead array was fixed at the same location, the microphone angles from the L1 and L2 nozzle exits were about one and two degrees larger than from the L0 nozzle. The top three lines are spectra at the set point 310 for L0 (k), L1 (c), and L2 (r) nozzles. The lines in the middle and bottom groups are for the set points 110 and 5000, respectively. The colors of lines are given by the letters in parentheses where k, c, r, b, g, and $\mathrm{m}$ correspond to black, cyan, red, blue, green, and magenta. The humps in the spectra between 20 and $40 \mathrm{KHz}$ are due to the protective grid caps on microphones.

At the high speed set point 310 , the effect of nozzle is clearly shown in the narrowband spectra. Those of the microphone number 5 show that the medium length nozzle L1 was quieter than the other nozzles in most of frequency range. In particular, the L1 nozzle was much quieter than the longest nozzle L0 in the frequency range higher than $4 \mathrm{KHz}$ and quieter than the shortest nozzle L2 for the frequencies higher than $10 \mathrm{KHz}$. The L0 nozzle was noisier in the frequency range between 4 and $13 \mathrm{KHz}$ and quieter between 13 and $22 \mathrm{KHz}$ compared to the L2 nozzle. Similar behavior was found in the spectra of the upstream angle microphones 10 and 13. However, the differences are not clearly shown in the downstream microphone number 1 . There was not any significant spectrum level difference between nozzles at the low speed set point 110 and the cold single jet condition set point 5000 .

The results of the classical beamforming analyses are presented in figures 4.2 to 4.4 for the 12CL mixer with the L0, L1, and L2 nozzles at the set point 310 . Figure 4.5 shows the results for the set points 5000, 110, and 310 in the top, middle and bottom rows, respectively. As mentioned before, the $\mathrm{x}$ coordinate was normalized by the nozzle diameter $(\mathrm{D}=7.245$ in.) and appropriately shifted so that the origin corresponds to the nozzle exit. The 
beamforming levels were computed along the jet centerline for all narrowband frequencies. At each frequency, the peak location and peak level were obtained at the axial location where maximum beamforming level occurs. The peak locations and peak levels were plotted in the first and second columns, where the results for the L0, L1, and L2 nozzles are plotted with black, cyan and red colors for the set points 5000 and 310 and with blue, green, and magenta colors for the set point 110. In order to show the effect of nozzle length on beamforming levels, the base 10 logarithms of beamforming levels for one nozzle are subtracted from those for the other nozzle. The difference between beamforming dB-levels is equal to the base 10 logarithms of the ratio of the beamforming levels. The contour plots of these beamforming dB-level differences between the L2 and L1, L0, and L1 and L2 and L0 nozzles are given in the last three columns with corresponding color bar in $\mathrm{dB}$ scale. Their maximum and minimum values are printed in the figures with corresponding frequencies and $\mathrm{x} / \mathrm{D}$.

As indicated in the narrowband spectra, the nozzle effect is strongly shown in figures 4.2 to 4.5 at the set point 310. For the medium length nozzle L1, the peak locations change from downstream positions to nozzle exit as frequencies are increased, except between 7 and $10 \mathrm{KHz}$ where the peak locations occur at the nozzle exit. The peak locations occur at about $0.5 \mathrm{D}$ rather than at the nozzle exit for the high frequencies between 10 and $20 \mathrm{KHz}$. In this beamforming analysis the frequencies higher than $25 \mathrm{KHz}$ are not successfully resolved as peak locations incorrectly appear at sidelobe positions. The peak locations of high frequencies above $4 \mathrm{KHz}$ for the longest nozzle $\mathrm{L} 0$ and above $8 \mathrm{KHz}$ for the shortest nozzle L2 occur at the nozzle exit. It indicates that this high frequency noise was generated either inside the nozzle or at the nozzle exit. The contour plots of differences of the logarithmic beamforming levels (or the ratios of the beamforming levels in $\mathrm{dB}$ scale) show that the largest differences between nozzles occur at the nozzle exit.

For the low frequencies up to $4 \mathrm{KHz}$ at the set point 310 , the peak locations gradually vary from downstream positions to about 1D distance from the nozzle exit as frequencies are increased. This low frequency noise was due to the jet flow and the peak locations occur some distances from the nozzle exit. The L1 nozzle has higher peak levels and more upstream peak locations compared to the other nozzles. The L0 and L2 nozzles have almost identical peak locations and levels.

At the low speed set point 110, the narrowband spectra in figure 4.1 and the peak locations and peak levels for different nozzles in figure 4.5 show almost identical results. However, the contour plots of beamforming dB-level differences reveal that longest nozzle L0 has higher noise level than the other two nozzles at the nozzle exit in the frequency range of 4 and $10 \mathrm{KHz}$ similar to the set point 310 case.

At the set point 5000 that mimics the unheated single cold jet condition, the effect of nozzle was not significant. The external hardware noise that appears at negative $\mathrm{x}$ locations has been exaggerated in the contour plots since the $\mathrm{x}$ coordinate origin shifts were dependent on the nozzle lengths.

\subsection{Effect of Nozzle on Confluent Splitter}

Figures 4.6 to 4.10 show the effect of nozzles on the Confluent splitter, Con. This axisymmetric splitter was used as a baseline for various mixer geometries. The linear array installed on the floor was used in this case. All 16 microphones were flush mounted without protective grid caps. Figure 4.6 shows the narrowband spectra from the microphone numbers $3,6,11$, and 16 whose microphone angles from the center of the L0 nozzle exit plane were $90^{\circ}, 100^{\circ}, 111^{\circ}$, and $128^{\circ}$, respectively. There was no L0 nozzle data at the set point 5000 .

The narrowband spectra in figure 4.6 and the peak levels in figure 4.10 show very little difference between nozzles. The L2 nozzle was slightly noisier than the others in the high frequency range at the set point 310 . However, figures 4.7 to 4.10 show that the peak locations were dependent on nozzles at this high speed set point 310. They occur at the nozzle exit for the frequencies higher than $4 \mathrm{KHz}$ for the $\mathrm{L} 2$ nozzle and higher than $8 \mathrm{KHz}$ for the L0 nozzle as in the low penetration mixer $12 \mathrm{CL}$ case. Although levels were much lower, the Confluent splitter shows similar nozzle effect as the low penetration mixer.

\subsection{Effect of Mixer on L1 Nozzle}

The Confluent splitter, two 12-lobed mixers, 12CL and 12UH, and two 20-lobed mixers 20UH and 20DS were tested with the L1 nozzle. As was shown in previous sections, the medium length nozzle L1 was the quietest among the three nozzles tested.

Figures 4.11 and 4.12 show the narrowband spectra and beamforming results from the floor array for the Confluent splitter and the 12-lobed high penetration mixer 12UH with the L1 nozzle. As shown in the spectra of the microphone number 11, the 12UH mixer reduced low frequency but increased high frequency noise with crossing frequencies at about 1.5 and $2 \mathrm{KHz}$ at the set points 110 and 310, respectively. The peak levels obtained from the classical beamforming method show the same behavior. The peak locations of the $12 \mathrm{UH}$ mixer occur at more 
upstream positions compared to the Confluent splitter for all frequencies. The contour plots of beamforming dBlevel difference show that the added high frequency noise of the 12UH mixer occurs at or close to the nozzle exit. This indicates that small scale turbulence enhanced by the mixer increased the level of this high frequency noise.

The narrowband spectra and the beamforming results are given in figures 4.13 and 4.14 where the L1 nozzle was tested with the $12 \mathrm{CL}, 12 \mathrm{UH}, 20 \mathrm{UH}$, and 20DS mixers by using the overhead array.

The comparison between the low and high penetration 12-lobed mixers, 12CL and 12UH shows that the spectrum levels of the 12UH mixer were a little lower than the 12CL mixer in low frequency ranges. However, when the frequencies were higher than 1 and $1.5 \mathrm{KHz}$ at the set points 110 and 310, respectively, the noise levels of the $12 \mathrm{UH}$ mixer was much higher than the 12CL mixer. In fact, in these high frequency ranges the 12-lobed mixer 12UH was noisier than the other 20-lobed mixers $20 \mathrm{UH}$ and 20DS of the same penetration. The contour plots of the beamforming dB-level differences reveal that the 12UH mixer produces much higher noise near the nozzle exit then the $12 \mathrm{CL}$ mixer for the frequencies higher than $500 \mathrm{~Hz}$ and $1 \mathrm{KHz}$ at the set points 110 and 310, respectively. It is shown in figure 4.14 that the $12 \mathrm{UH}$ mixer has the most upstream peak locations and highest peak levels among all four mixers.

By comparing the results of the 20 and $12 \mathrm{UH}$ mixers, we can show that high frequency noise can be reduced by reducing the width of lobe or by increasing lobe number. The 20-lobed 20UH mixer has lower spectrum levels than the 12-lobed 12 $\mathrm{UH}$ mixer in the high frequency range above about 1 or $1.5 \mathrm{KHz}$. The low frequency noise of the 20UH mixer was slightly increased. The spectrum levels of the $20 \mathrm{UH}$ mixer were in between those of the $12 \mathrm{UH}$ and $12 \mathrm{CL}$ mixers. Figure 4.14 shows that most of the peak level reductions between the 20 and $12 \mathrm{UH}$ mixers occur in the frequency range between 1 and $6 \mathrm{KHz}$. The contour plots of beamforming dB-level differences between 12UH and $20 \mathrm{UH}, 12 \mathrm{UH}, 12 \mathrm{CL}, 20 \mathrm{UH}$, and $12 \mathrm{CL}$ show that the most noise reduction occurs near the nozzle exit.

Introducing a deep scallop was very successful in reducing mixer noise. The narrowband spectrum levels of the 20-lobed high penetration mixer with deep scallop, 20DS were lower than the 20 and 12UH mixers in all frequencies at both set points 110 and 310. The spectrum levels from 112 degree microphone show that the 20DS mixer was much quieter than the low penetration mixer 12CL in the low frequency ranges below $5 \mathrm{and} 10 \mathrm{KHz}$ at the 110 and 310 set points, respectively. The penalty at higher frequency was relatively small especially at the high speed set point 310 . The contour plots in figure 4.14 confirm that the 20DS mixer was quieter than the $12 \mathrm{UH}$ and 20UH mixers in almost all grid points that cover from -1D to 7D streamwise locations and from $200 \mathrm{~Hz}$ to $50 \mathrm{KHz}$ frequencies. The 20DS mixer was also quieter than the 12CL mixer in downstream locations and low frequencies, though it was a little noisier at higher frequencies near the nozzle exit.

\subsection{Effect of Mixer on L2 Nozzle}

The L2 nozzle was the shortest one tested. The previous results show that this was noisier than the L1 nozzle especially near the nozzle exit. The narrowband spectra and beamforming results of the Confluent splitter and the $12 \mathrm{CL}$ and $12 \mathrm{UH}$ mixers from the floor array are given in 4.15 and 4.17 .

The general trends were similar to the L1 nozzle case discussed in the previous section. For example, the spectrum level from the $111^{\circ}$ floor microphone in figure 4.15 shows that the level of the $12 \mathrm{UH}$ mixer was higher than the Confluent splitter for the frequencies higher than $1.5 \mathrm{KHz}$.

Difference between this and the quietest L1 nozzle occurs at high frequencies where the former was noisier than the latter. The contour plots in figure 4.16 show that, at the frequencies higher than about 6 or $10 \mathrm{KHz}$ at the high speed set point 310 , the L2 nozzle with high penetration mixer, 12UH was much noisier at the nozzle exit than with the low penetration mixer 12CL.

\subsection{Effect of Mixer on L0 Nozzle}

It is shown in the previous section that the longest L0 nozzle generates higher high frequency noise at the nozzle exit compared to the L1 nozzle.

Figures 4.18 to 4.20 show the narrowband spectra and beamforming results from the overhead array for the $12 \mathrm{CL}, 12 \mathrm{UH}, 12 \mathrm{UM}$, and Confluent splitter. The noise level of the medium penetration mixer 12UM was in between those of the 12CL and 12UH mixers. In general, at all set points 5000, 110, and 310, the noise level of the 12UM mixer was lower than the $12 \mathrm{UH}$ mixer in high frequency range and lower than the $12 \mathrm{CL}$ mixer in low frequencies. The spectra from $112^{\circ}$ microphone, in figure 4.18 show that this medium penetration mixer has the highest spectrum level at about $10 \mathrm{KHz}$ at the set point 310 . 


\section{Beamforming Results From the Azimuthal Array at Set Point 310}

In this section, the results of the azimuthal phased-array analyses will be presented for the Confluent splitter and the12CL mixer at the set point 310 . The phased-array results will be compared with the data from the Particle Image Velocimetry (PIV) tests by Bridges and Wernet (2004).

The azimuthal phased-array data was processed at nine frequencies from 1 to $9 \mathrm{KHz}$. As mentioned before, the "as measured" narrowband cross-spectral densities with the bandwidth of $12.21 \mathrm{~Hz}$ were used for the classical beamforming method. The beamforming levels are presented at the streamwise positions of the microphones, i.e., $\mathrm{x} / \mathrm{D}=0,0.37$ and 0.75 for the L0, L1, and L2 nozzles, respectively. As expected, there was no major difference between the beamforming results computed at the nozzle exit and at the microphone locations except a slight level change.

The beamforming analysis from the linear phased-array in the previous section, as in figures 4.2, 4.7, 4.19, and 4.20, shows that the peak noise locations for both the 12CL mixer and the Confluent splitter with the L0 nozzle occur at the nozzle exit for frequencies higher than $4 \mathrm{KHz}$. The contour plots in figure 4.2 indicate that this high frequency noise was very strongly concentrated at the nozzle exit for the 12CL mixer. Meanwhile, it was less strongly concentrated for the Confluent splitter as shown in figures 4.7 and 4.20.

The azimuthal array beamforming results of the 12CL mixer and the Confluent splitter with the L0 nozzle at the high speed set point 310 are given in figure 5.1. The beamforming levels were computed in the $y-z$ plane at $x=0$ where the azimuthal-array microphones were located. In this L0 nozzle case, all microphones were located at a $90^{\circ}$ angle relative to the nozzle exit. The white circle represents the nozzle exit. The narrowband spectra of the microphone 6, plotted in the bottom-right, show that the 12CL mixer enhances the high frequency noise for the frequencies higher than about $1.5 \mathrm{KHz}$. The beamforming levels of the 12CL mixer at $6006 \mathrm{~Hz}$, plotted in the topleft in figure 5.1, clearly show 12 red spots that are associated with the locations of the 12 lobes. It is also interesting to note that the core flow region was stronger noise source than the bypass flow region. Meanwhile, the beamforming levels of the Confluent splitter show that the outer shear layer at the nozzle lip and the core region were the noisy area. The ratio of the beamforming level of the $12 \mathrm{CL}$ mixer to the Confluent splitter, as shown in the bottom left figure in $\mathrm{dB}$ scale, indicates that the increased noise level of the 12CL mixer relative to the Confluent splitter was mainly due to the core-flow and lobe-induced noise.

The beamforming results at other frequencies from 1 to $9 \mathrm{KHz}$ are given in figures 5.2 to 5.4. The locations of 12 lobes of the $12 \mathrm{CL}$ mixer are very well detected as strong noise source areas for frequencies $5 \mathrm{KHz}$ and higher as shown in figure 5.2. The core-flow also produces strong noise in all frequencies for this $12 \mathrm{CL}$ mixer. At $9 \mathrm{KHz}$, the outer-shear-layer noise level becomes large and three distinctive noise spots, core-flow region, lobe region, and the outer shear layer are identifiable. The beamforming levels of the Confluent splitter in figure 5.3 show that the outer shear layer was the most important noise source in all frequencies. The beamforming resolution was not sufficient for the long waves at 1 to $2 \mathrm{KHz}$. There are some frequencies where the center becomes red. It is interesting to note that the thickness of the red ring at the outer shear layer becomes very thin at $9 \mathrm{KHz}$. The ratio of the beamforming levels plotted in figure 5.4 confirms that the enhanced high frequency noise compared to the Confluent splitter was mainly due to the core-flow and lobe-area noise.

Figures 5.5 and 5.6 show the axial mean velocity and the axial rms turbulence velocity measured by using the PIV method by Bridges and Wernet (2004) for the 12CL mixer and the Confluent splitter with the L0 nozzle at the axial location of $x / D=0.2$. The flow condition of set point 312 of the PIV test was the same as the 310 condition except there was a free jet of Mach number 0.2 . This free jet would not have significantly impacted the turbulence inside the nozzle. In general, the phased-array results were consistent with the PIV data. There was a good correlation between the high turbulence regions from the PIV tests and the high noise source regions from the phased-array measurements. The turbulence velocity of the 12CL mixer shows higher levels in the core flow region, the lobe region and the thin outer shear layer. The CFD results by Garrison et al (2005) show similar results although the highest turbulence level occurs in the very thin outer shear layer. The PIV data of the Confluent splitter shows high turbulence velocities in the core-bypass mixing layer and the outer shear layer. The phased-array results from the $90^{\circ}$ azimuthal array, however, show that the outer shear layer was a more important noise source.

The beamforming contour plots of the 12CL mixer with the L2 nozzle in figure 5.7 are similar to those of the L0 nozzle in figure 5.2. They show strong a noise source in the core-flow region and also show red spots associated with the lobes. The contour plots of the Confluent splitter in figure 5.8 are also similar to the L0 case in figure 5.3. The red ring at the outer shear layer of the Confluent splitter with the L2 nozzle at $9 \mathrm{KHz}$ becomes thicker than the L0 nozzle case.

The difference between the 12CL mixer and the Confluent splitter for the L1 nozzle was not as strong as the L0 nozzle case as shown in figures 5.10 to 5.12. The 12CL mixer, however, still shows the strong core flow noise. 
The ratios of the beamforming levels between the $\mathrm{L} 0$ and $\mathrm{L} 1$ nozzles for the $12 \mathrm{CL}$ mixer are plotted in figure 5.13 and between the L2 and L1 nozzles are given in figure 5.14. The beamforming level ratios are also plotted in figures 5.15 and 5.16 for the Confluent splitter. The PIV results by Bridges and Wernet (2004) for the 12CL mixer with the L0, L1, and L2 nozzles at the axial location of $\mathrm{x} / \mathrm{D}=0.2$ and set point 312 are given in figure 5.17. The beamforming level ratios for the 12CL mixer in figures 5.13 and 5.14 confirm the results that the core flow and the lobe area are strong noise source for the L0 and L2 nozzles.

\section{Concluding Remarks}

The phased-array system has been successfully applied to identify the noise source locations. It was shown that the 16 microphone linear phased-array system installed in parallel with the jet-flow centerline and the 32 microphone azimuthal phased-array system installed at the nozzle exit could detect correct noise source locations.

In general, the high frequency jet noise was coming from near the nozzle exit and the low frequency noise was coming from near the end of the potential core. The source locations are smoothly varying from the nozzle exit to the end of potential core as the frequencies decrease at the flow condition 5000 that mimics single flow jet. The frequency-source location dependency was not smooth at the other flow conditions because mixer and nozzle altered the flow field from that of a single flow jet. The various mixers and nozzles increase/decrease noise of certain frequency components and the phased-array beamforming can find the locations of the added noise sources.

For all mixers, Confluent splitter and 12CL, 12UH, 20UH, and 20DS mixers, the largest differences of the beamforming levels between nozzles occur at the nozzle exit. The high frequency noise, higher than 3 or $4 \mathrm{KHz}$, was strongly dependent on the nozzle geometry. Among the three nozzles tested, the medium length nozzle L1 was the quietest for all mixers. The nozzle effect is stronger at the high speed set point 310 than at the low speed set point 110 .

Introducing a deep scallop was very successful in reducing mixer noise. The narrowband spectrum levels of the 20-lobed high penetration mixer with deep scallop, 20DS are lower than the 20 and 12UH mixers in all frequencies at both set points 110 and 310 .

The beamforming analyses from the 32 microphone azimuthal phased-array for the $12 \mathrm{CL}$ mixer at the set point 310 show that the core flow and the lobe area are strong noise sources for the L0 and L2 nozzles. The 12 noisy spots associated with the lobe positions of the 12CL mixer with the L0 nozzle are very well detected for frequencies $5 \mathrm{KHz}$ and higher. Meanwhile, the beamforming levels of the Confluent splitter show that the outer shear layer was the most important noise source in most cases. In general, there was a good correlation between the high turbulence regions from the PIV tests and the high noise source regions from the phased-array measurements.

\section{References}

1. Mengle, V.G., Baker, V.D., and Dalton, W.N., Lobed mixer Design fro Noise Suppression, NASA/CR—2002210823, 2002.

2. Tester, B.J., Fisher, M.J., and Dalton, W.N., A contribution to the understanding and prediction of jet noise generation in forced mixers, AIAA-2004-2897.

3. Bridges, J. and Wernet, M.P., Cross-stream PIV measurements of jets with Internal Lobed Mixers, AIAA2004-2896.

4. Dougherty, R.P., Beamforming in acoustic testing, in Aeroacoustic Measurements, Mueller, T.J. (ed.), 2002.

5. Underbrink, J.R., Aeroacoustic phased array testing in low speed wind tunnel, in Aeroacoustic Measurements, Mueller, T.J. (ed.), 2002.

6. Johnson, P. and Dudgeon, D., Array signal processing: Concepts and techniques, Prentice Hall, New Jersey, 1993.

7. Lee, S.S., Phased-array study of jets with various internal mixers and nozzles, NASA/CR-2005-213870.

8. Garrison, L.A., Lyrintzis, A.S., Blaisdell, G.A., and Dalton, W.N., Computational fluid dynamics analysis of jets with internal forced mixers, AIAA-2005-2887.

9. Garrison, L.A., Dalton, W.N., Lyrintzis, A.S., and Blaisdell, G.A., On the development of semi-empirical noise models for the prediction of the noise from jets with forced mixers, AIAA-2004-2898.

10. Tester, B.J. and Fisher, M.J., A contribution to the understanding and prediction of jet noise generation in forced mixers: Part II flight effects, AIAA-2005-3094. 


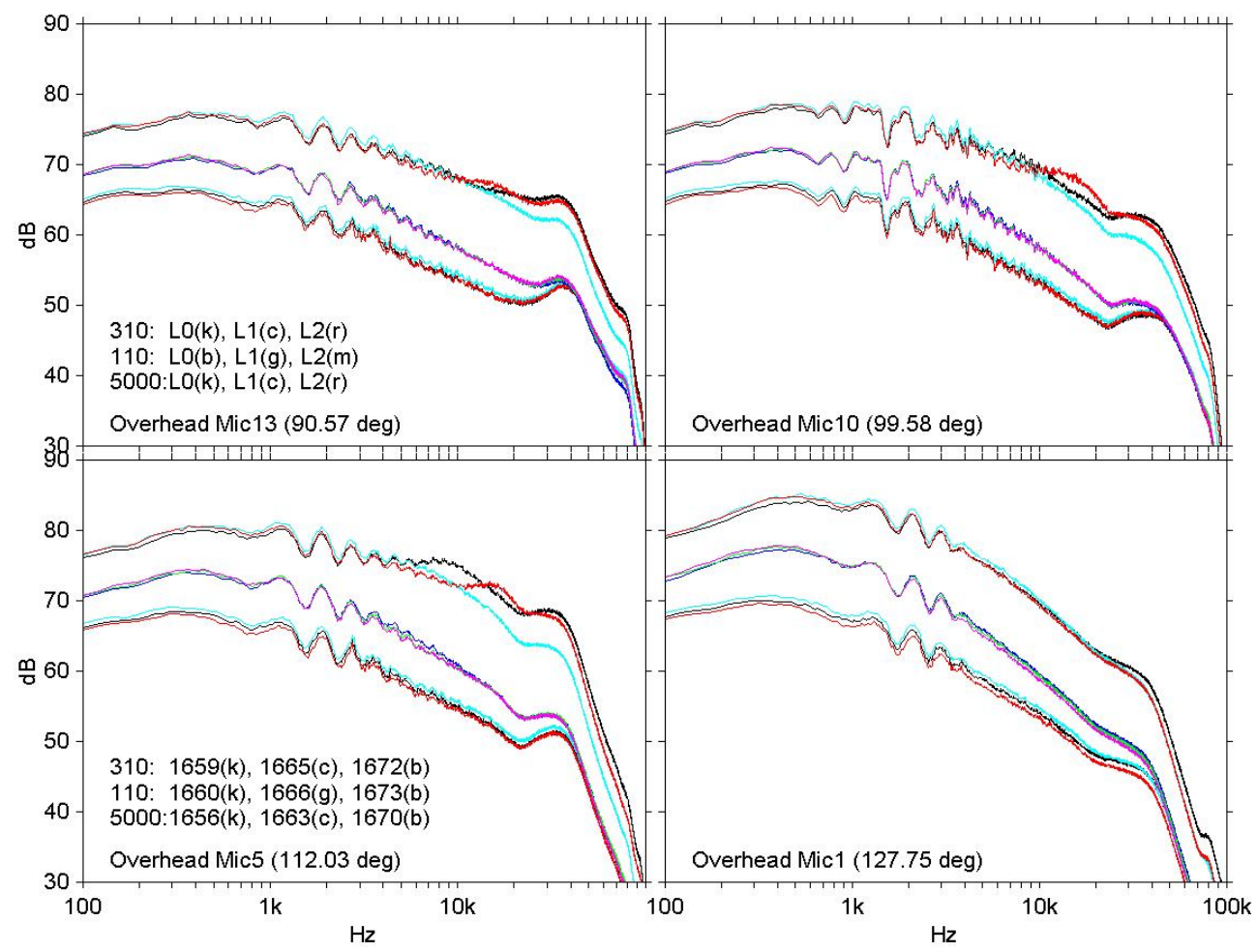

Figure 4.1.-Narrowband spectra of 12CL mixer with L0, L1, and L2 nozzles from overhead linear array at set points 5000, 110, and 310 (Escort numbers 1656, 1663, 1670, 1660, 1666, $1673,1659,1665$, and 1672).
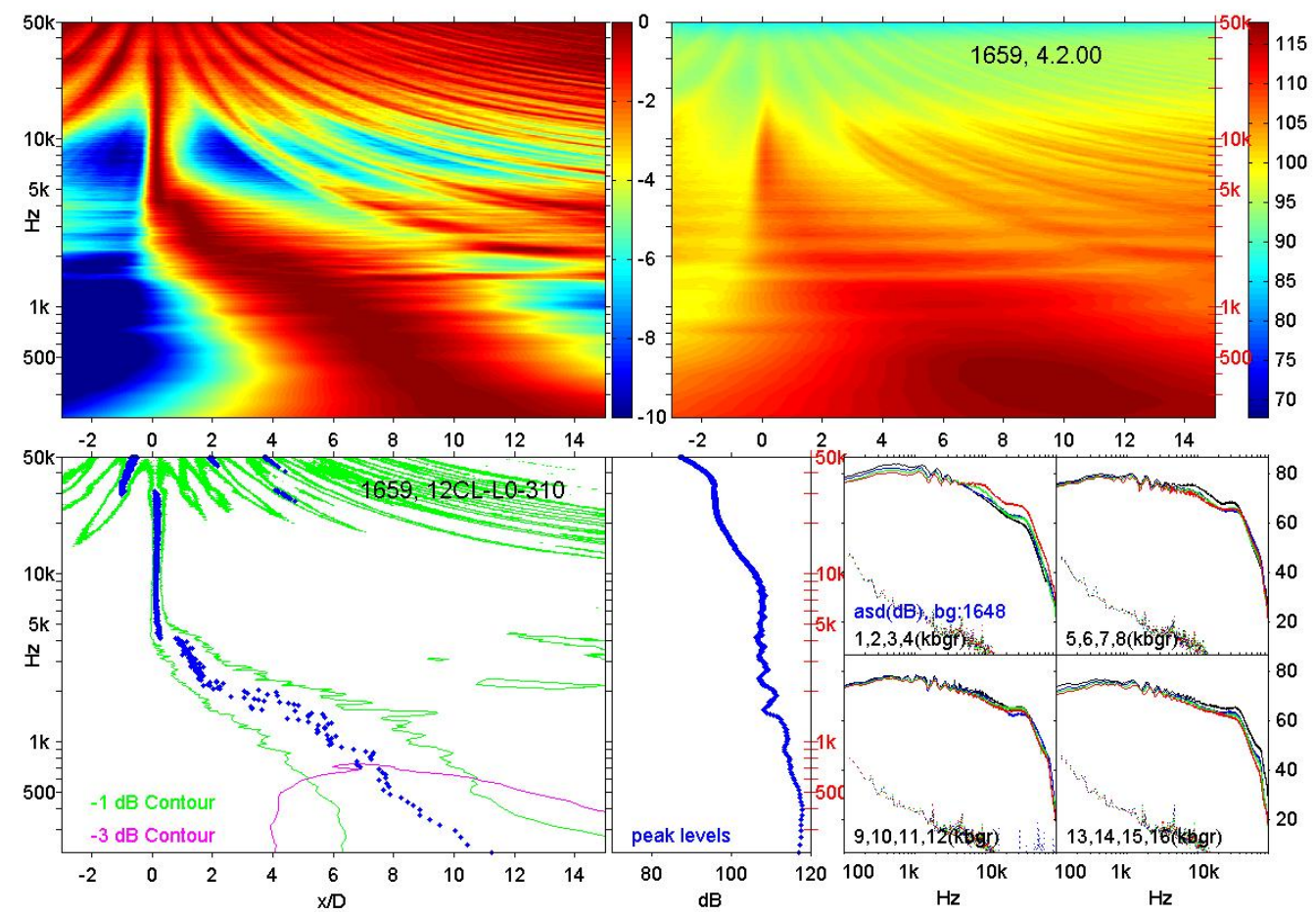

Figure 4.2.-Classical beamforming results from overhead linear array for 12CL mixer with L0 nozzle at set point 310 (Escort number 1659). 

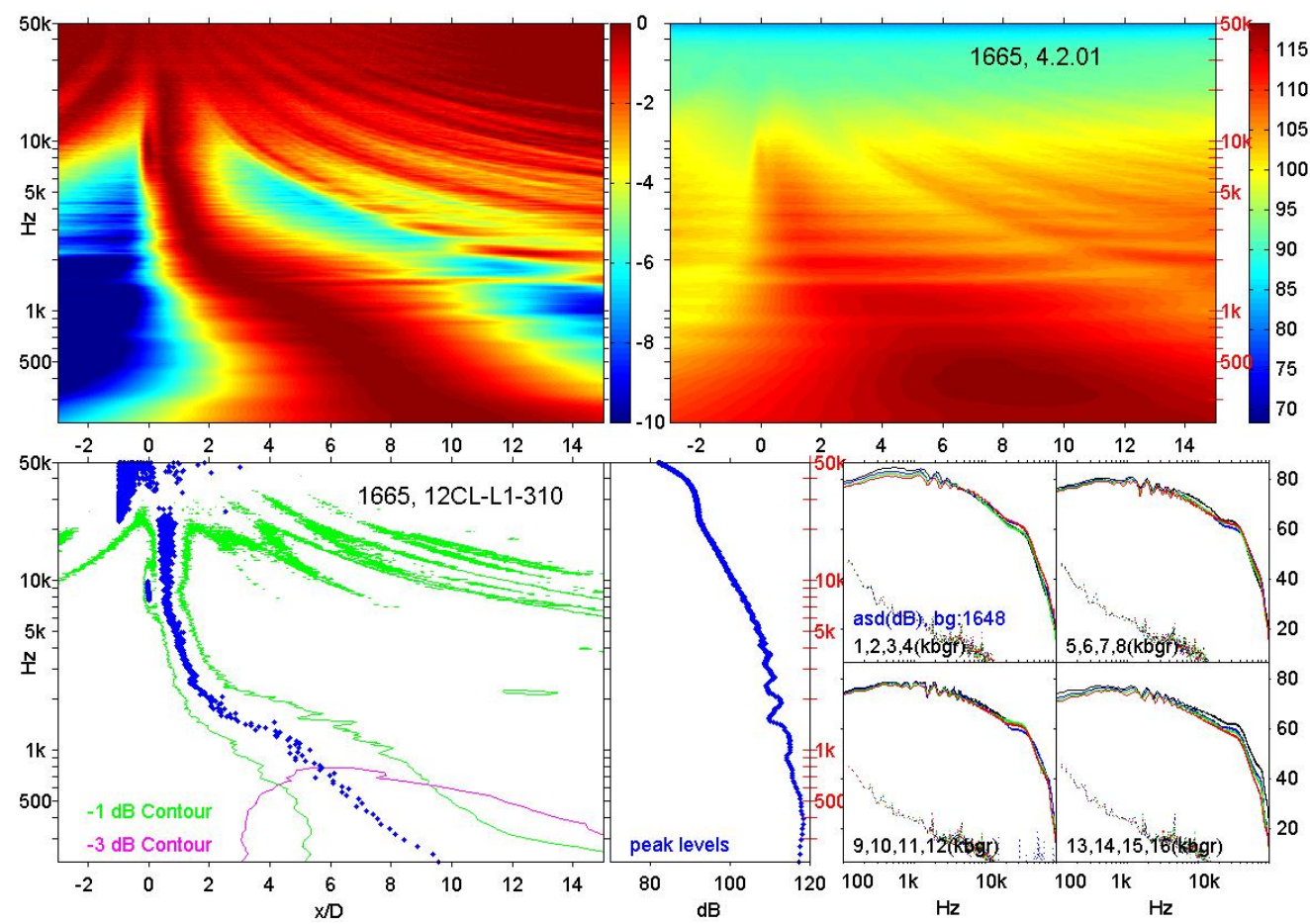

Figure 4.3.-Classical beamforming results from overhead linear array for 12CL mixer with L1 nozzle at set point 310 (Escort number 1665).
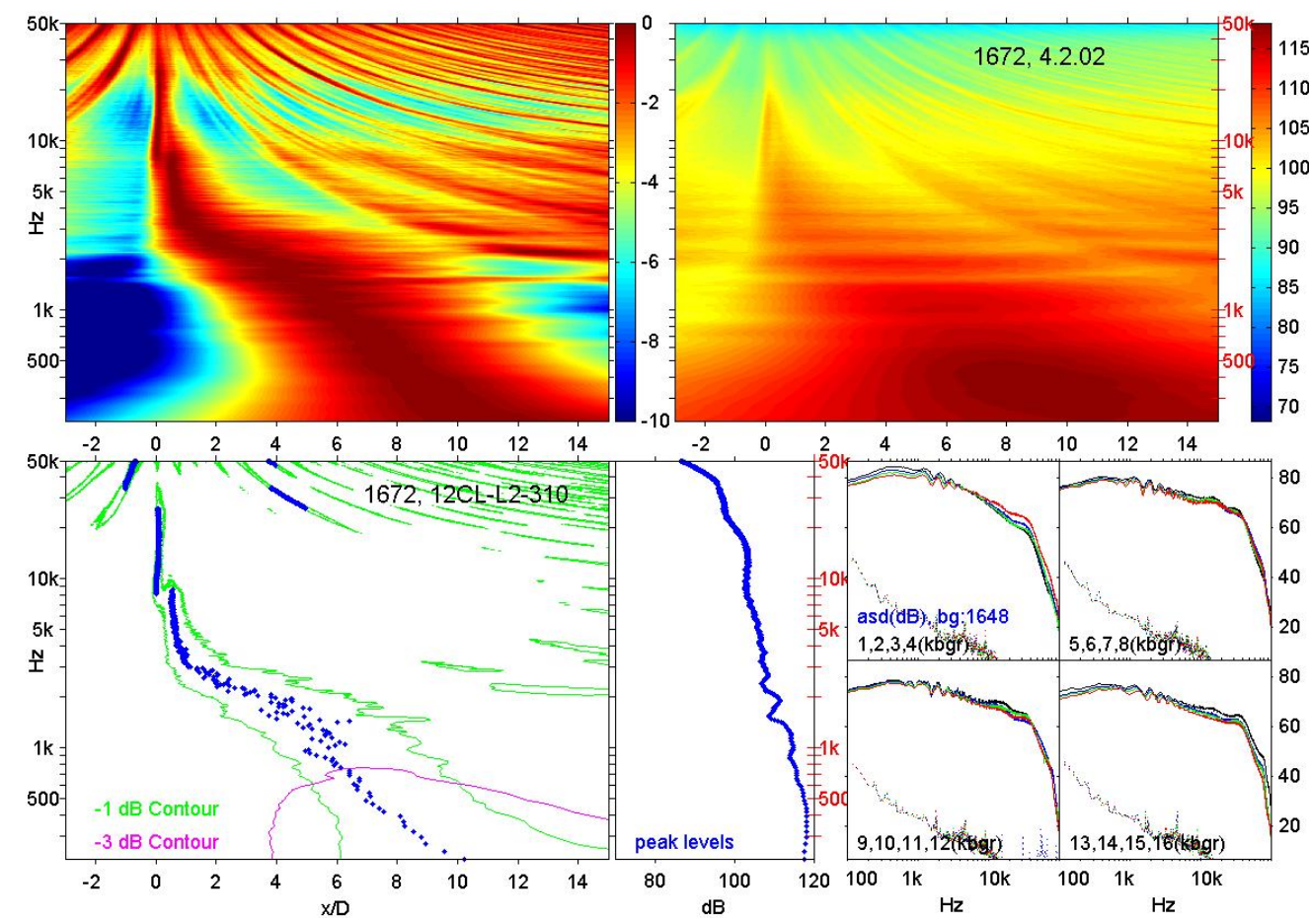

Figure 4.4.-Classical beamforming results from overhead linear array for 12CL mixer with L2 nozzle at set point 310 (Escort number 1672). 

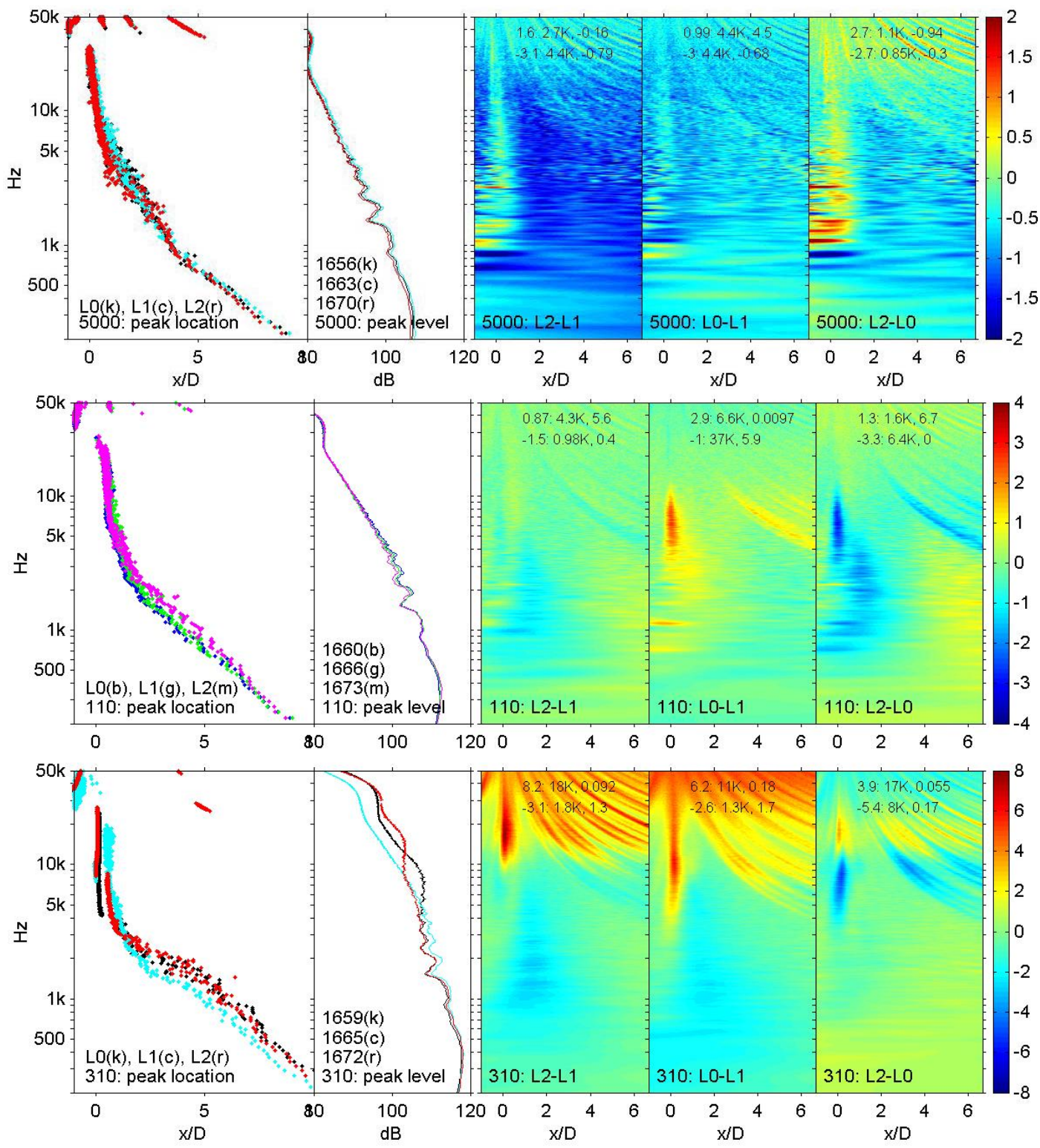

Figure 4.5.-Beamforming results of 12CL mixer with L0, L1 and L2 nozzles. 


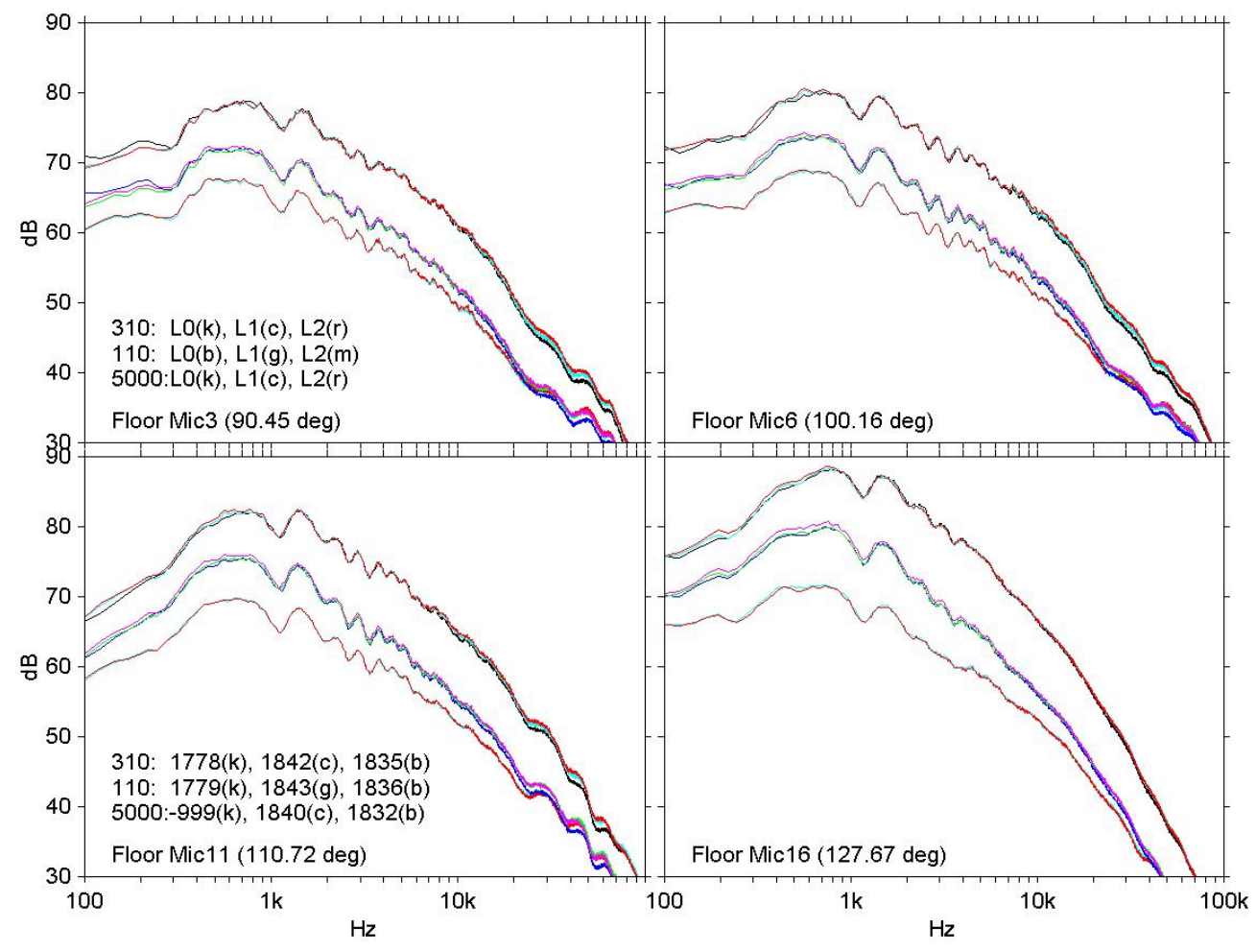

Figure 4.6.- Narrowband spectra of Confluent splitter with L0, L1, and L2 nozzles from floor linear array at set points 5000, 110, and 310 (Escort numbers 1840, 1832, 1779, 1843, 1836, 1778,1842 , and 1835).
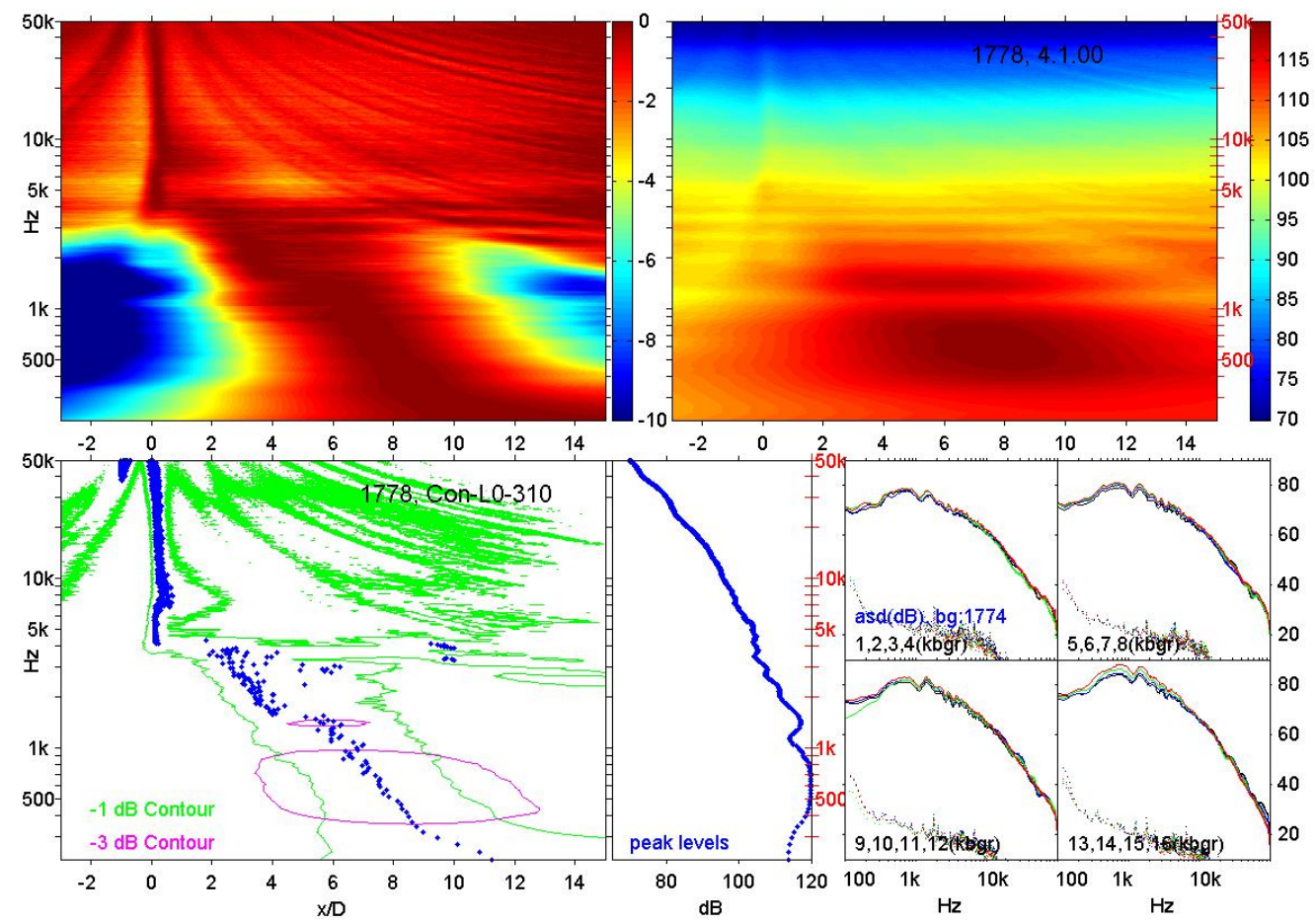

Figure 4.7.-Classical beamforming results from floor linear array for Confluent splitter with L0 nozzle at set point 310 (Escort number 1778). 

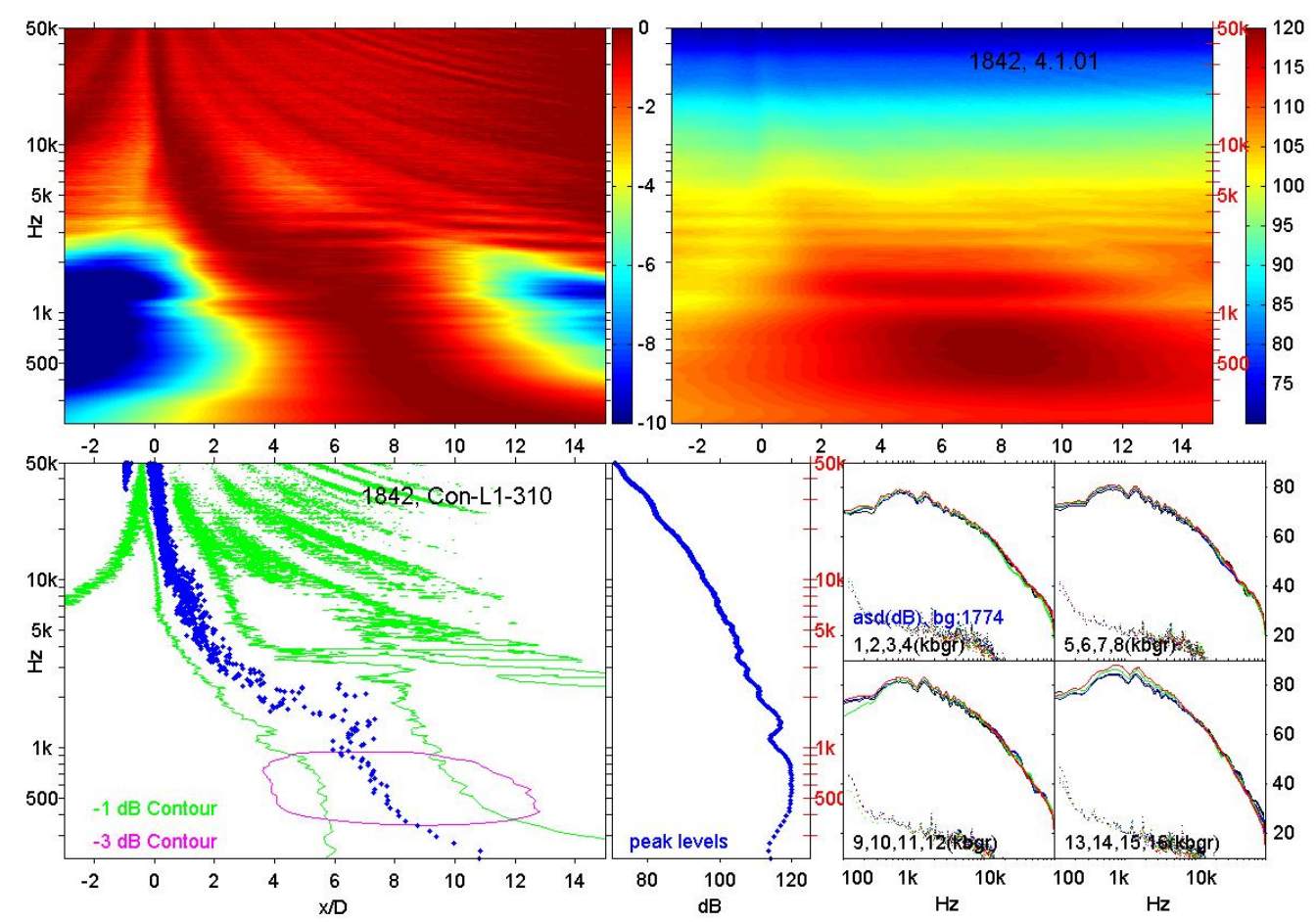

Figure 4.8.-Classical beamforming results from floor linear array for Confluent splitter with L1 nozzle at set point 310 (Escort number 1842).
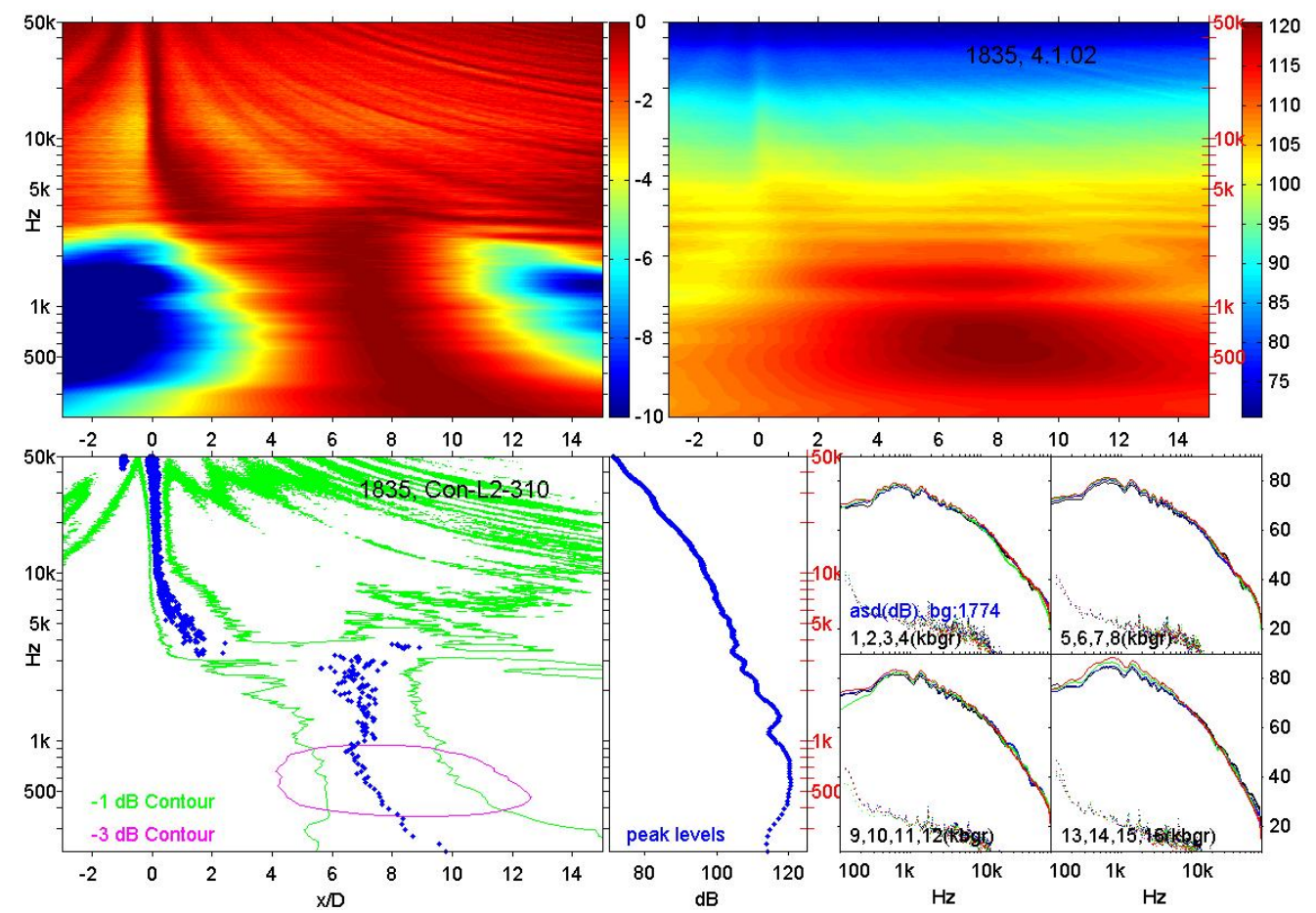

Figure 4.9.-Classical beamforming results from floor linear array for Confluent splitter with L2 nozzle at set point 310 (Escort number 1835). 

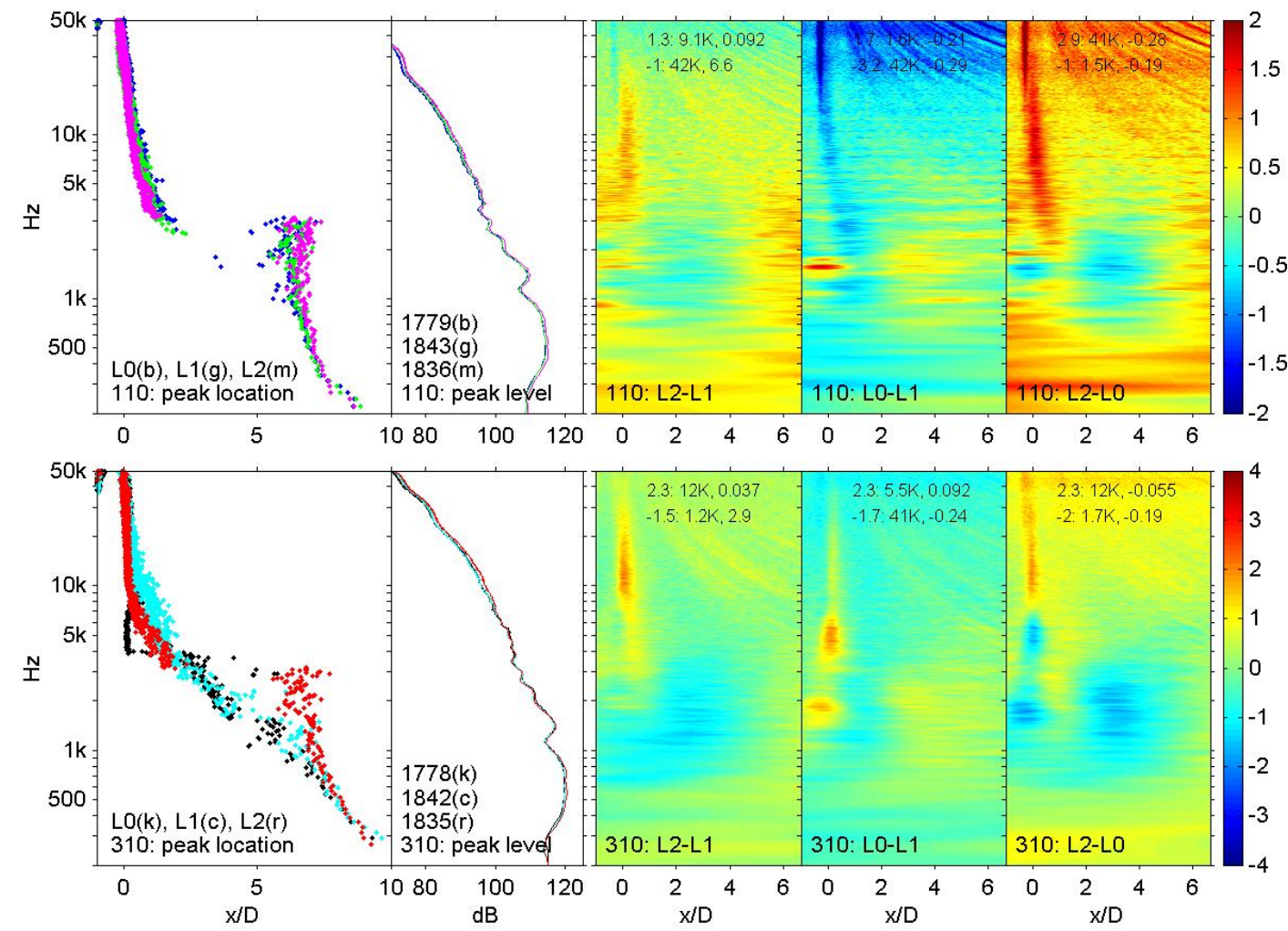

Figure 4.10.-Beamforming results of Confluent splitter with L0, L1, and L2 nozzles. 


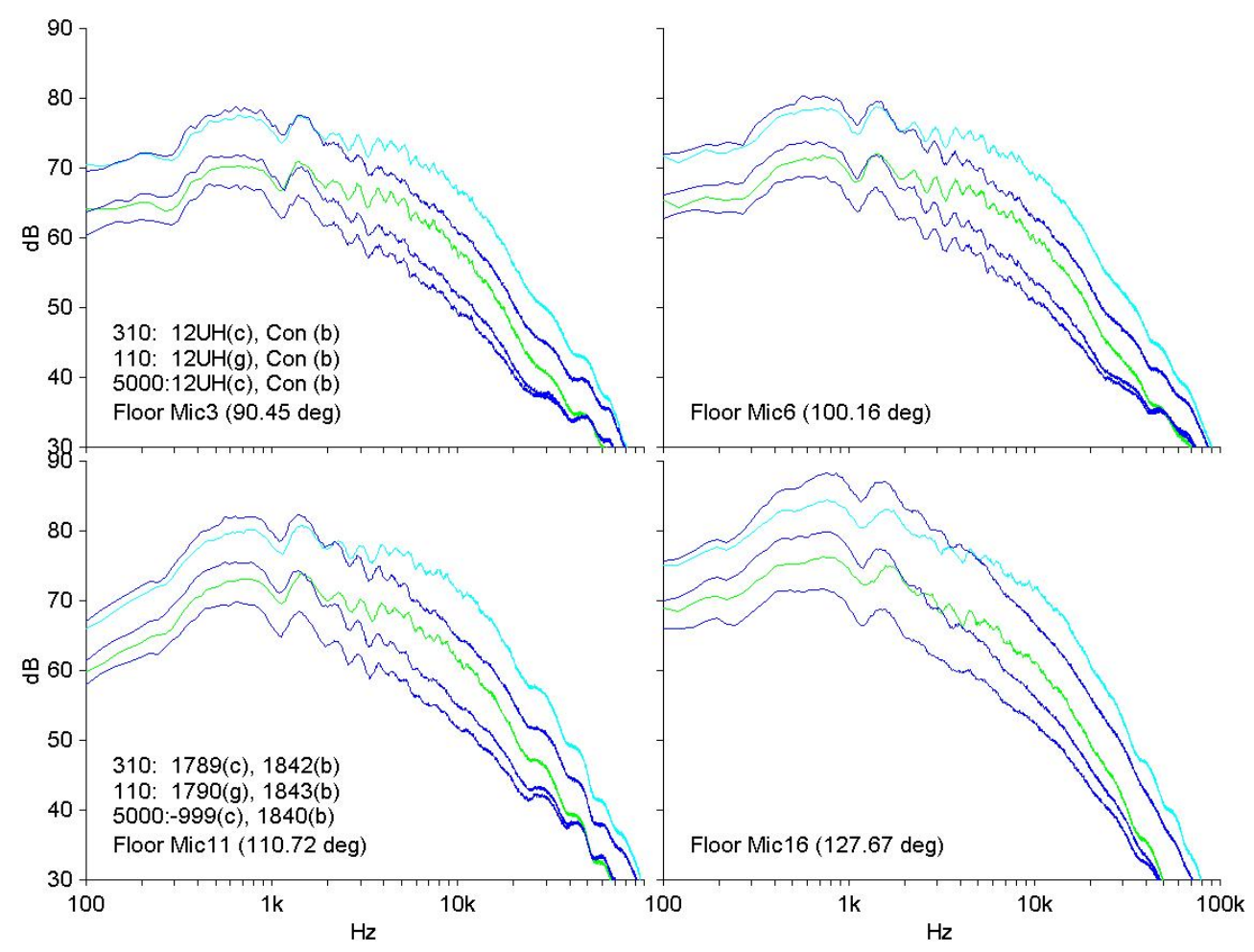

Figure 4.11.- Narrowband spectra of L1 nozzle with Confluent splitter and 12UH mixer from floor linear array at set points 5000, 110 and 310 (Escort numbers 1840, 1790, 1843,1789 and 1842).
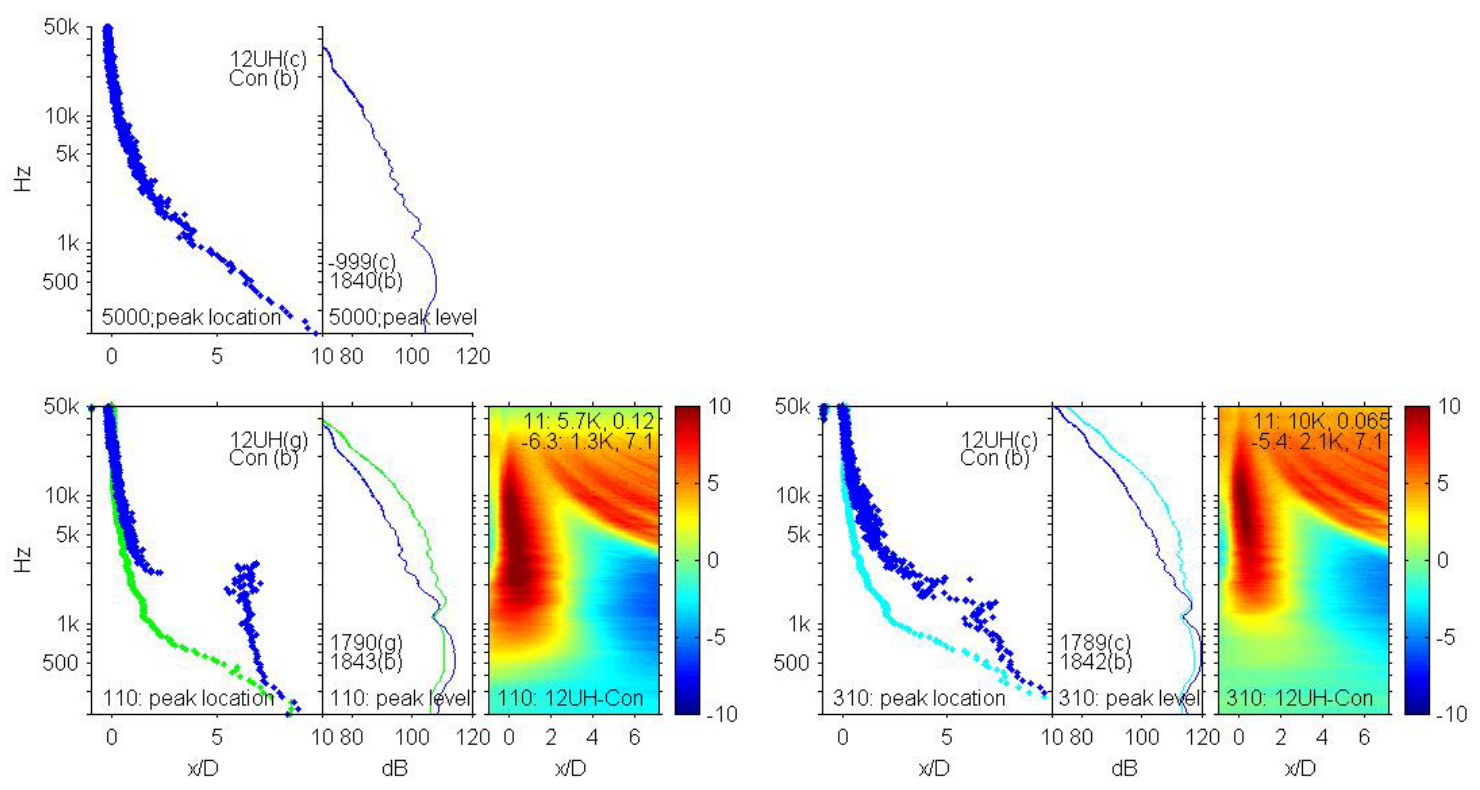

Figure 4.12.-Beamforming results of L1 nozzle with Confluent splitter and 12UH mixer. 


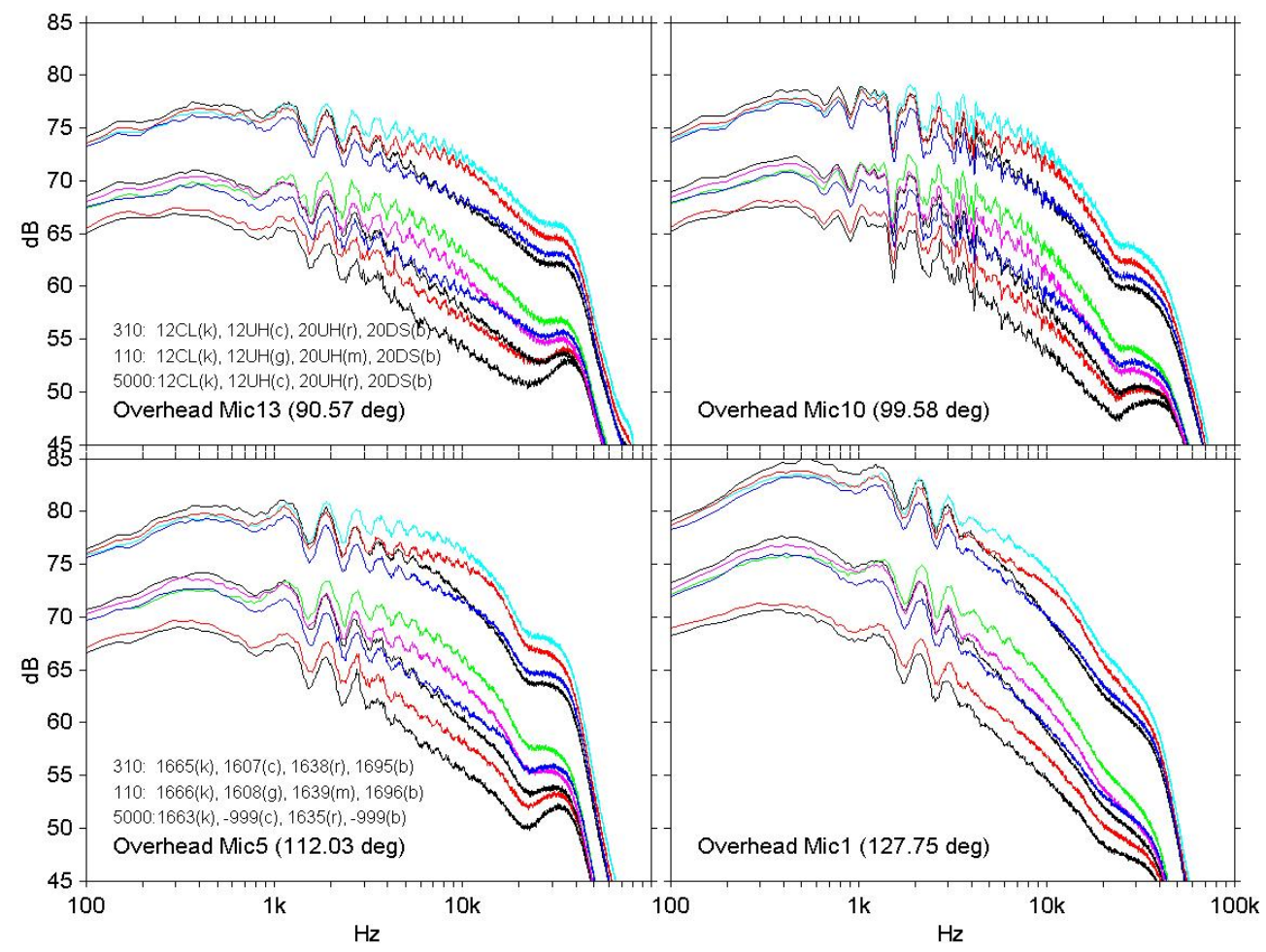

Figure 4.13.-Narrowband spectra of L1 nozzle with 12CL, 12UH, 20UH, and 20DS mixers from overhead linear array at set points 5000, 110, and 310 (Escort numbers 1663, 1635, $1666,1608,1639,1696,1665,1607,1638$, and 1695).
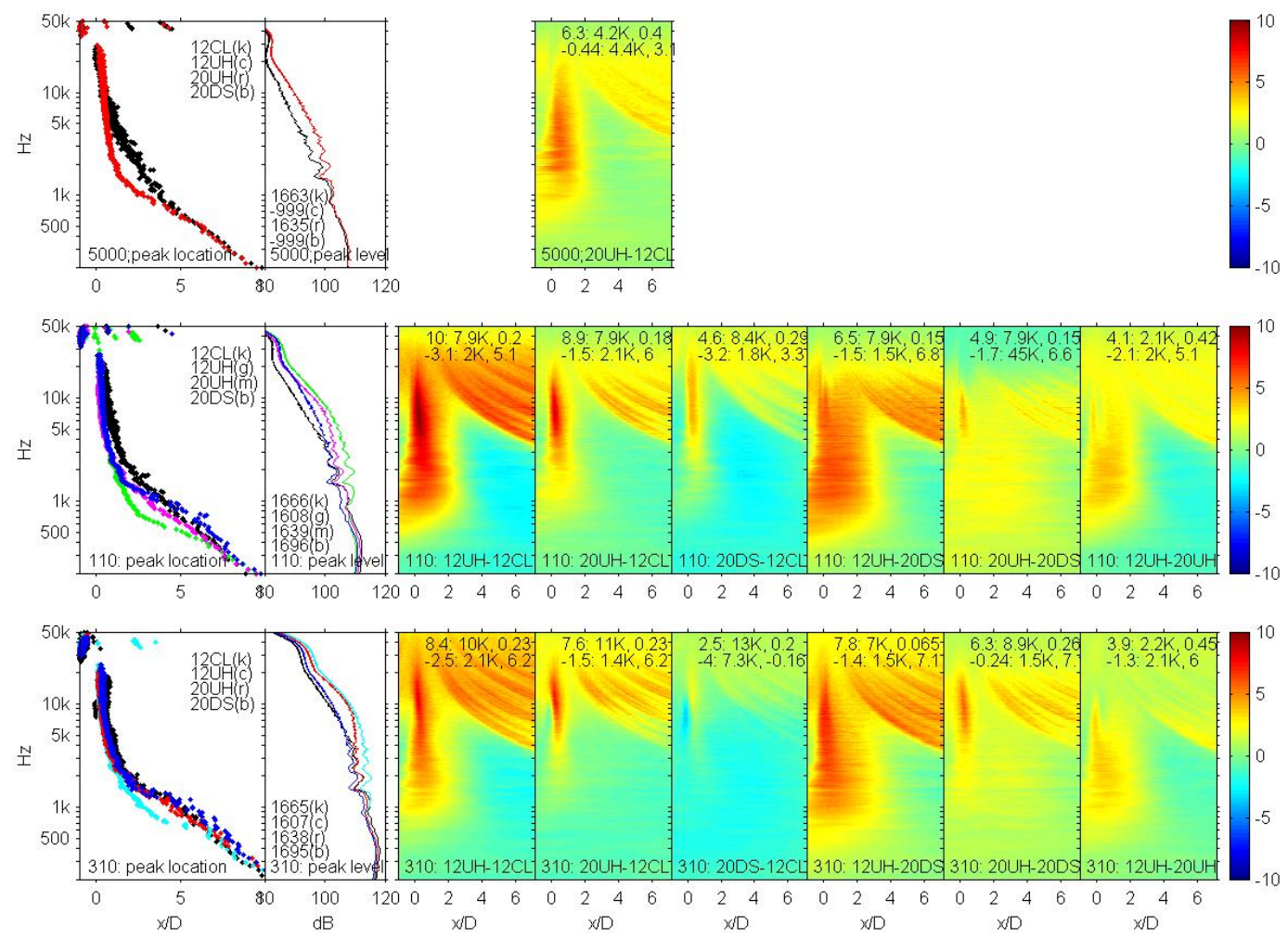

Figure 4.14.-Beamforming results of L1 nozzle with 12CL, 12UH, 20UH, and 20DS mixers. 


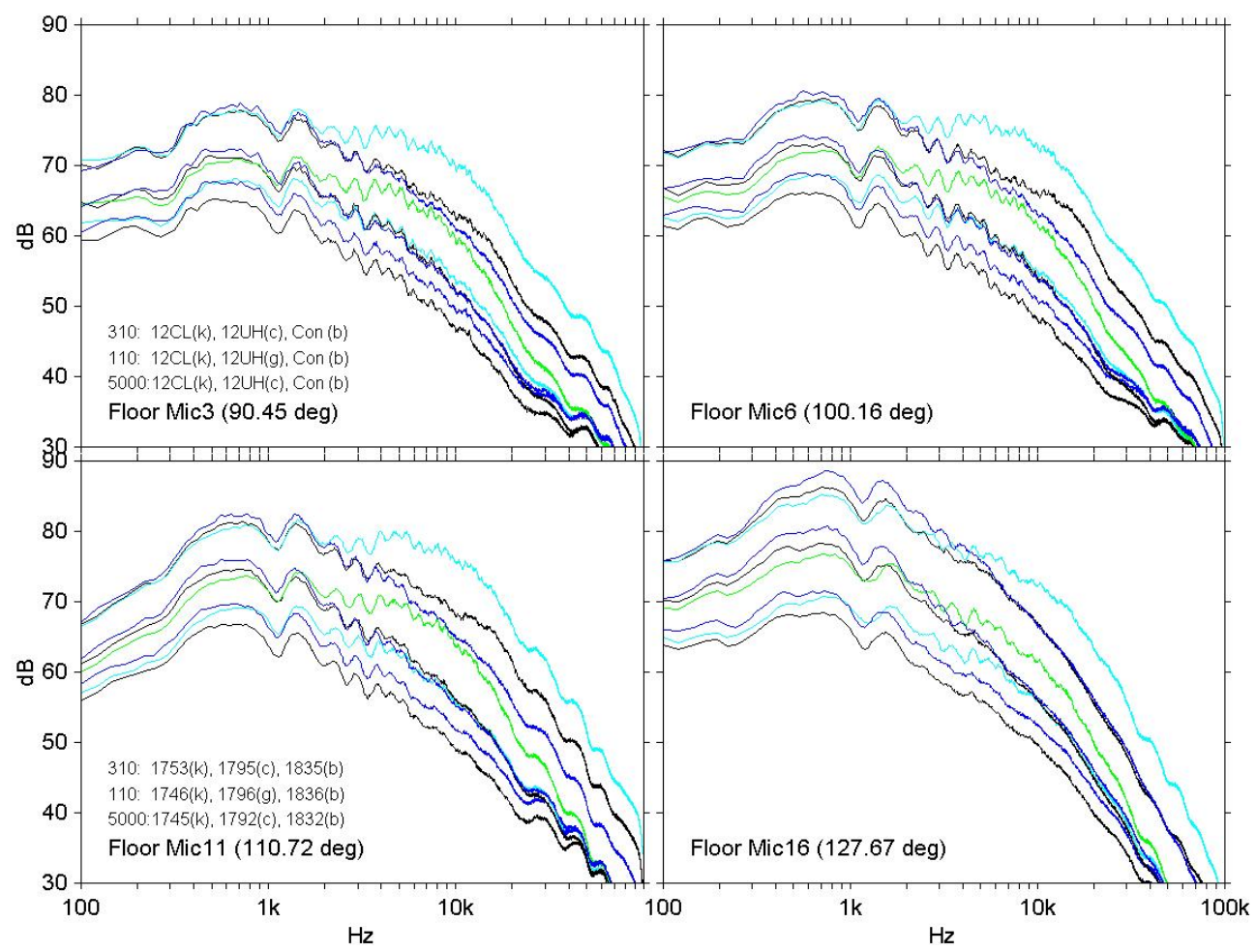

Figure 4.15.- Narrowband spectra of L2 nozzle with Con, 12CL and 12UH mixers.
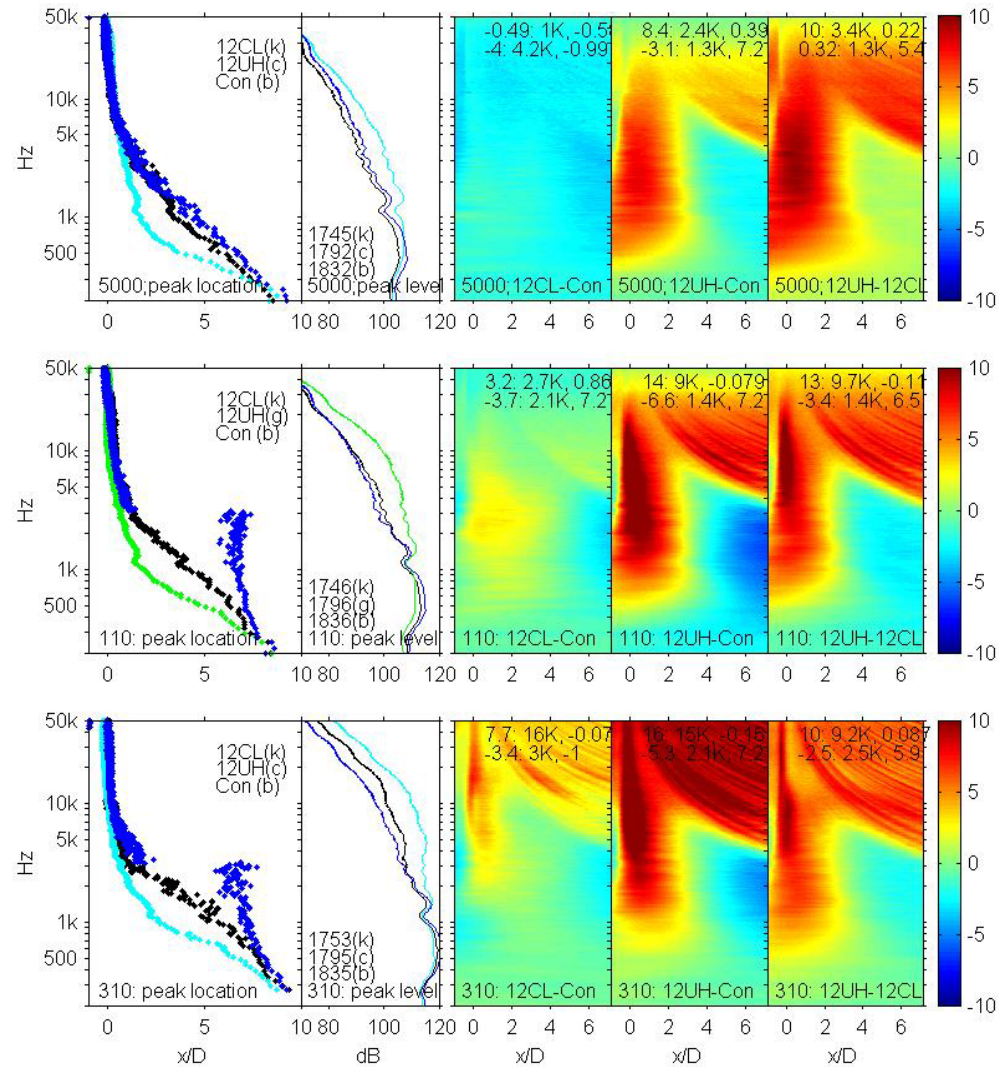

Figure 4.16.-Beamforming results of L2 nozzle with Con, 12CL and 12UH mixers. 


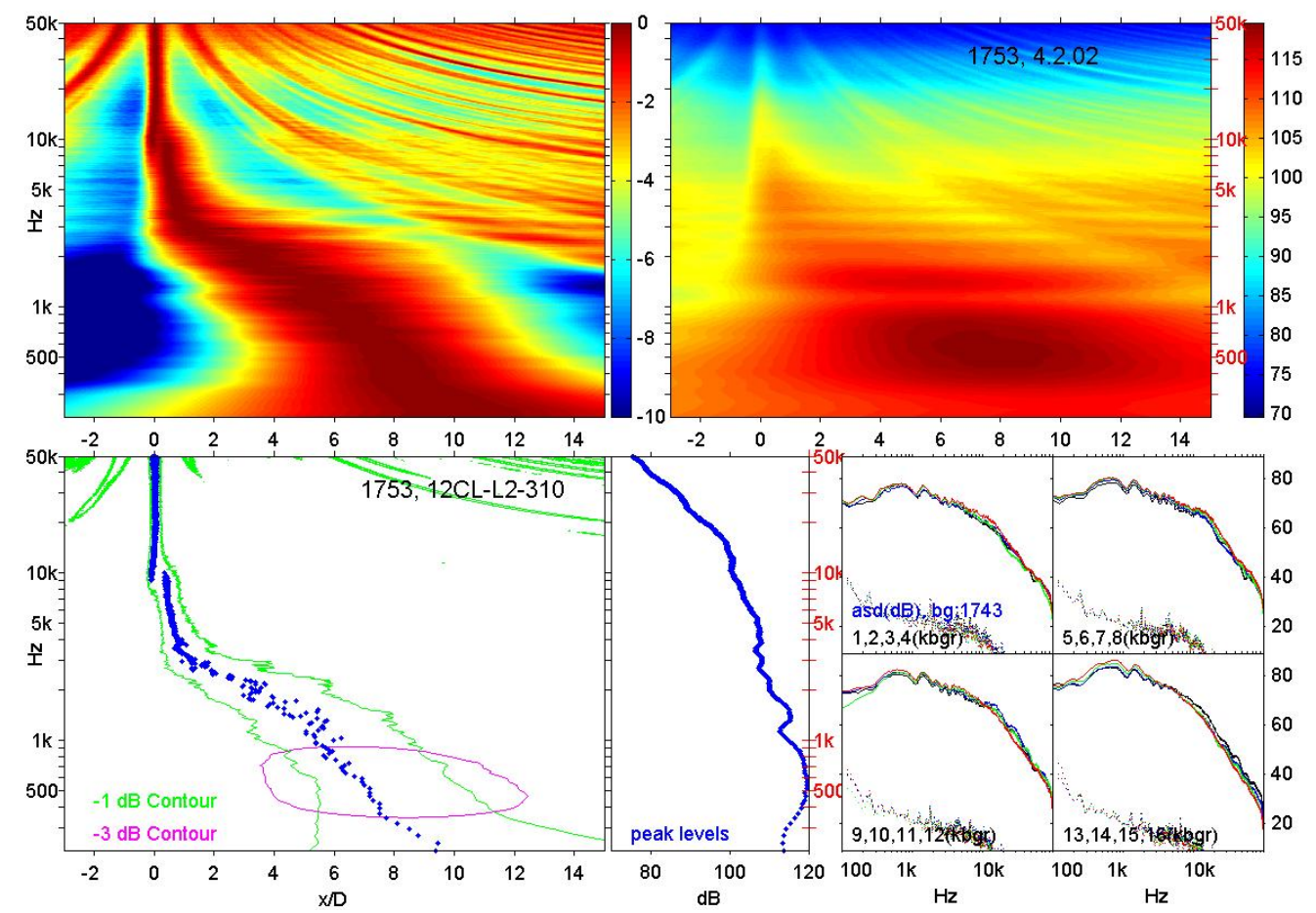

Figure 4.17.-Classical beamforming results from floor linear array for $12 \mathrm{CL}$ mixer with L2 nozzle at set point 310 (Escort number 1753). 


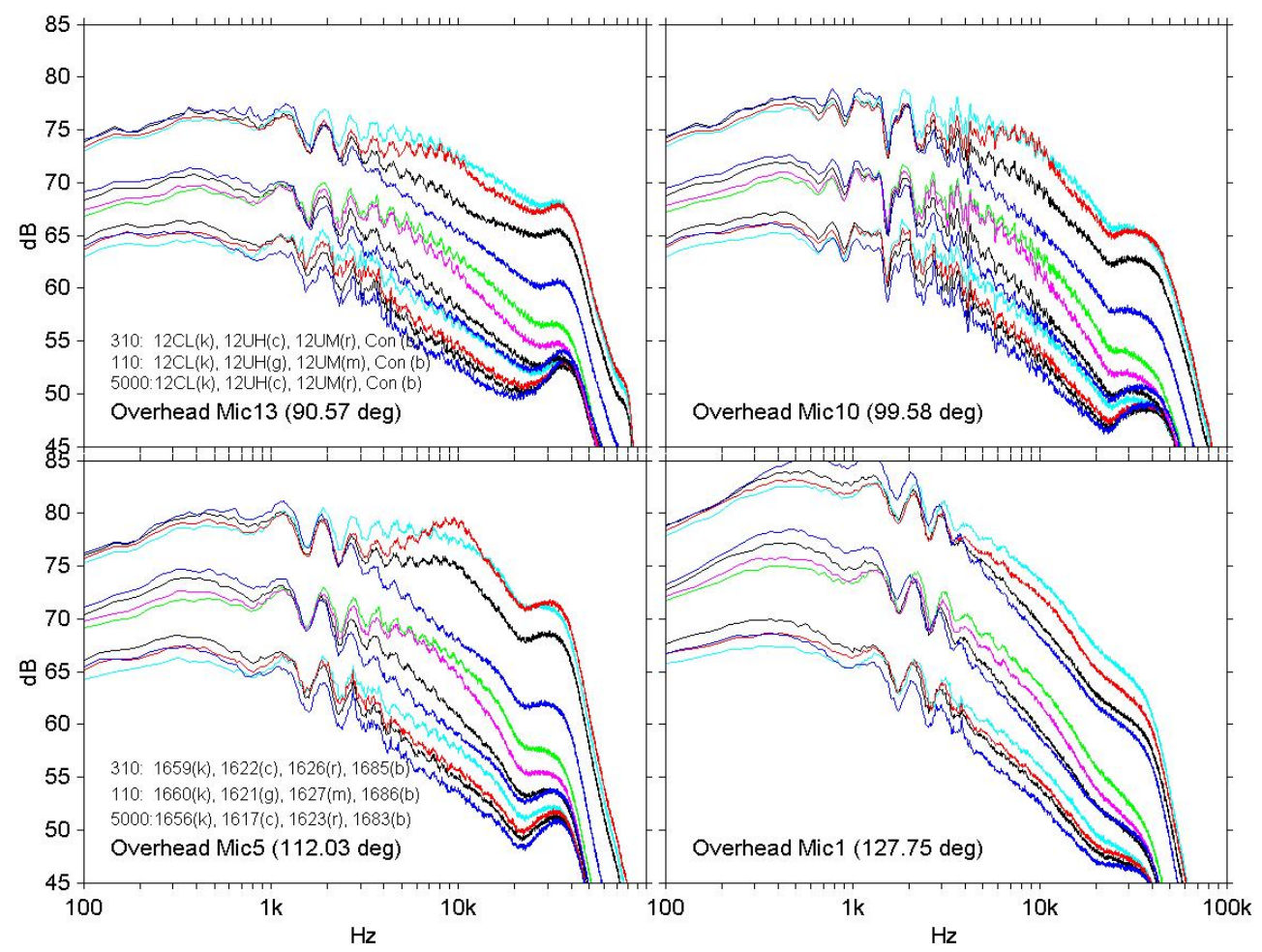

Figure 4.18.-Narrowband spectra of L0 nozzle with 12CL, 12UH, 12UM, and Con mixers.
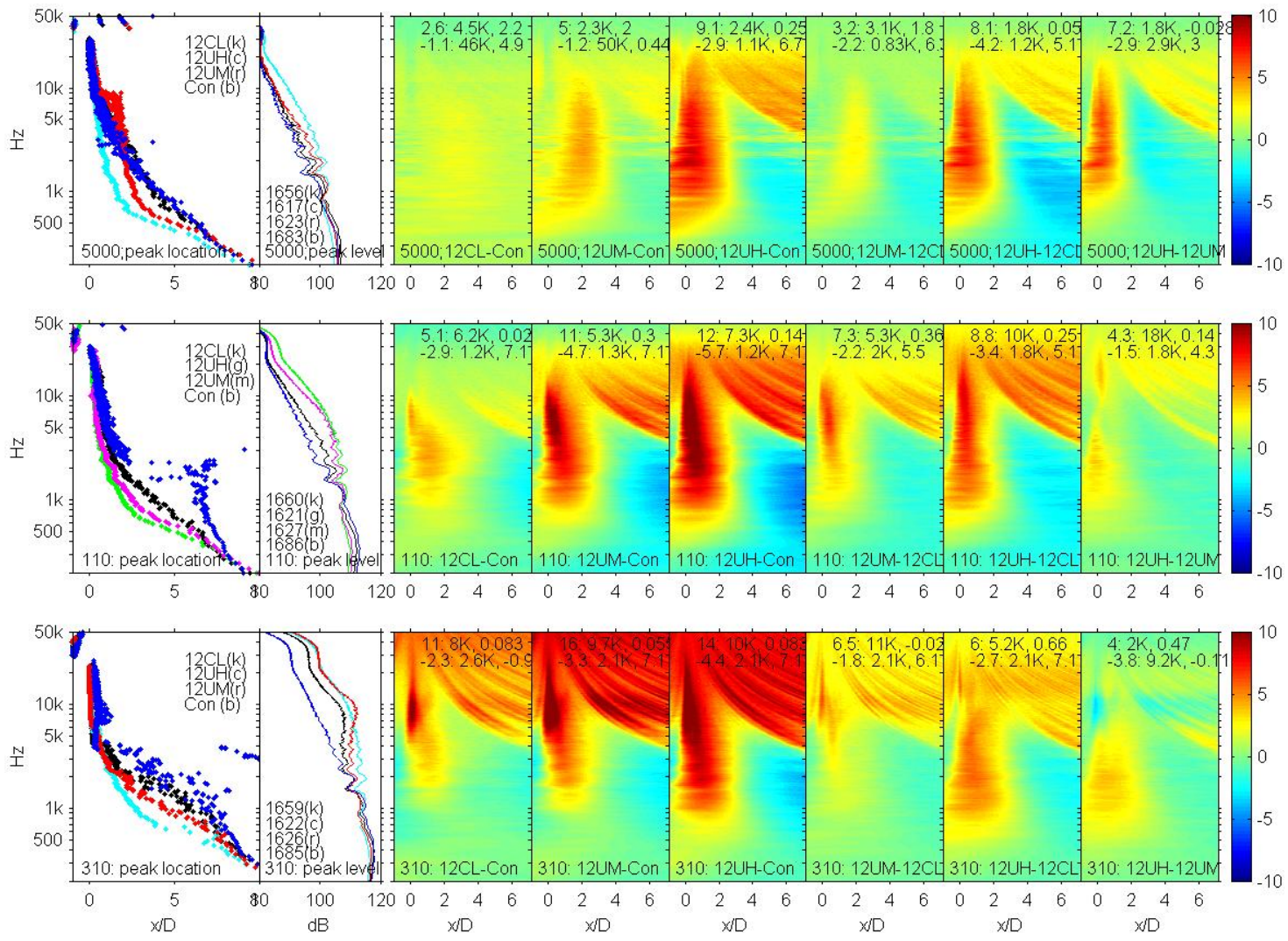

Figure 4.19.-Beamforming results of L0 nozzle with 12CL, 12UH, 12UM, and Con mixers. 


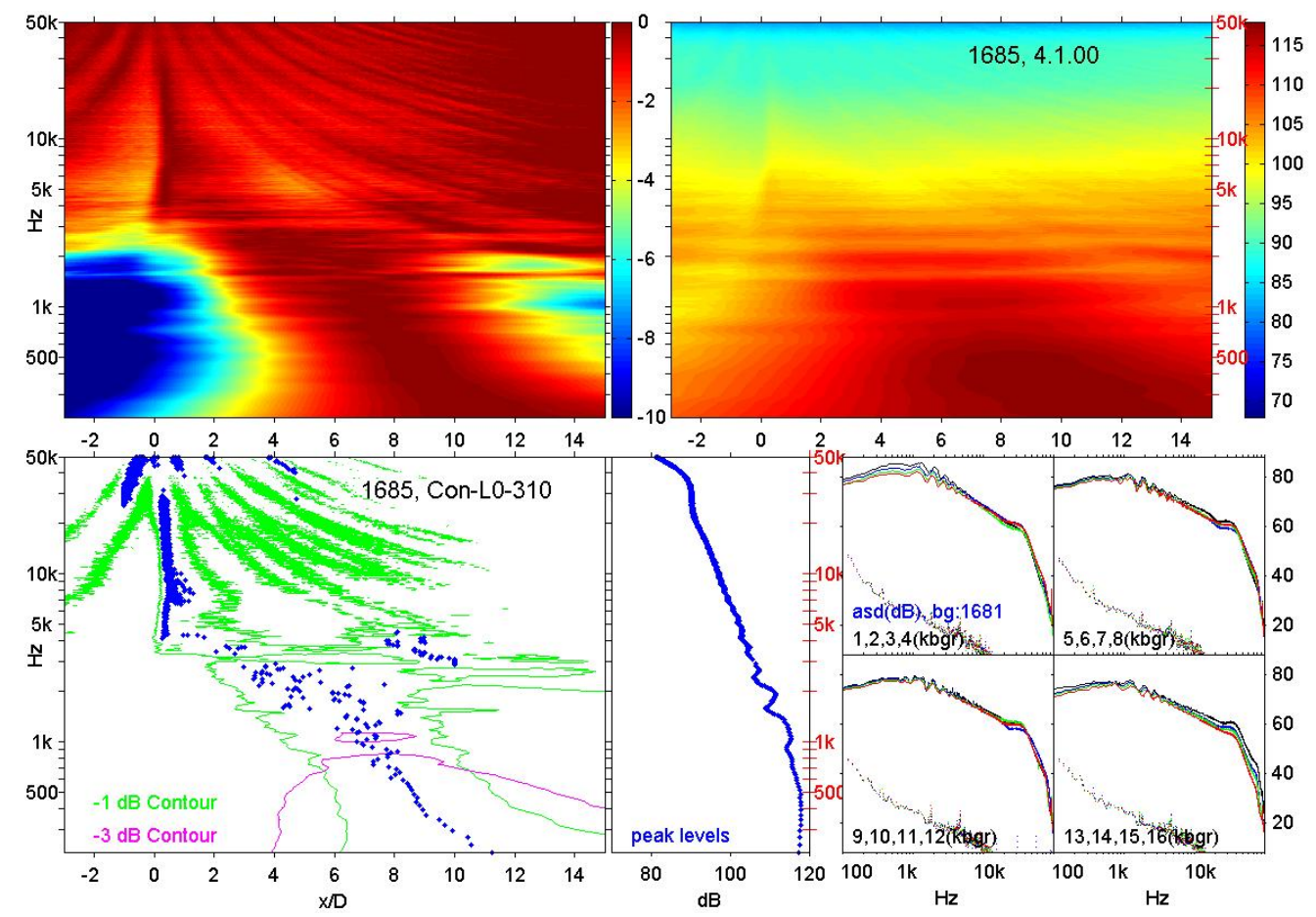

Figure 4.20.-Classical beamforming results from overhead linear array for Confluent splitter with L0 nozzle at set point 310 (Escort number 1685). 

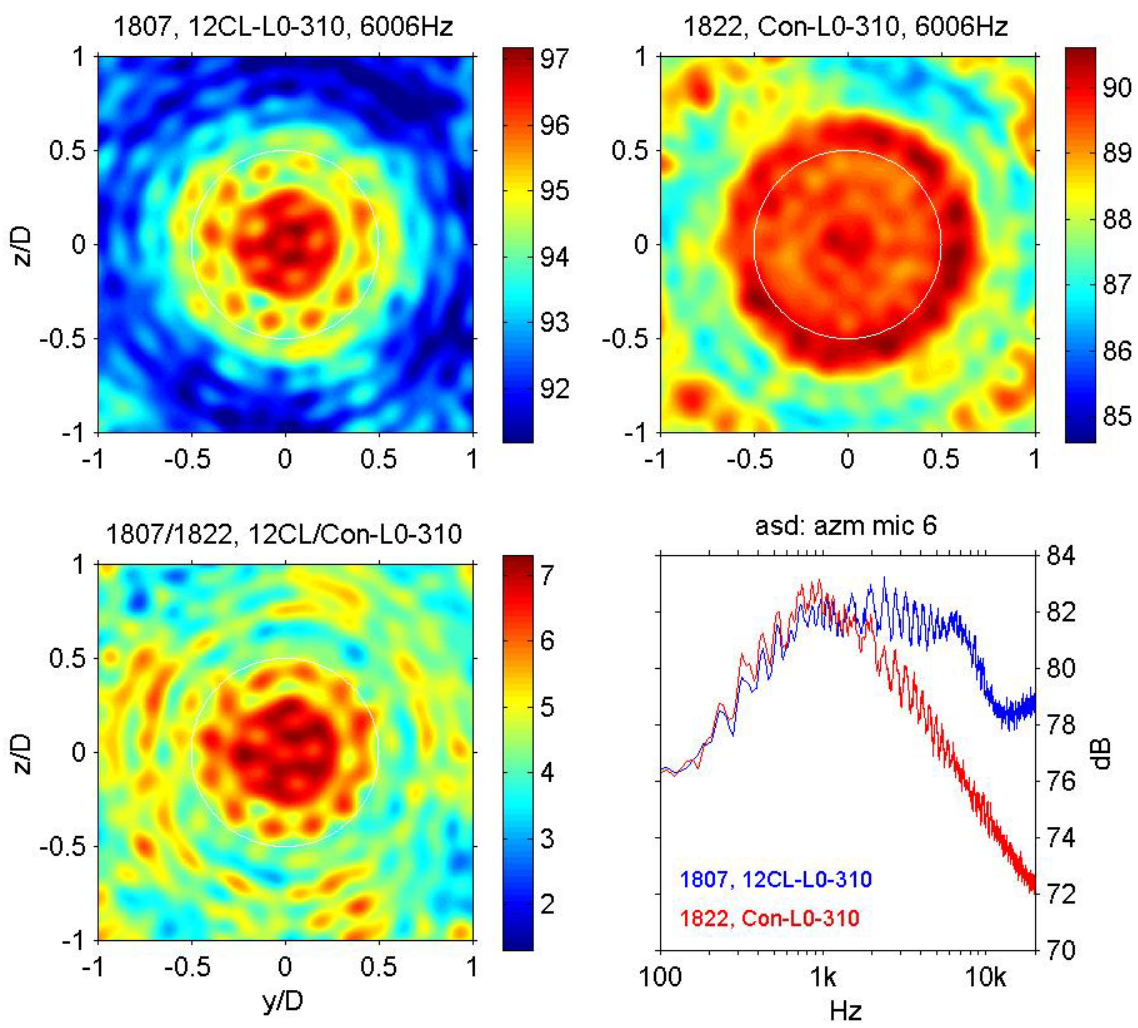

Figure 5.1.-Azimuthal array beamforming levels at $6006 \mathrm{~Hz}$ for $\mathrm{L} 0$ nozzle, set point 310 , and $\mathrm{x}=0$ : $12 \mathrm{CL}$ mixer (upper left), Confluent splitter (upper right) and the ratio of the beamforming level of 12CL mixer to Confluent splitter (lower left), along with narrowband spectra of microphone 6 (lower right). Escort numbers: 1807 and 1822.
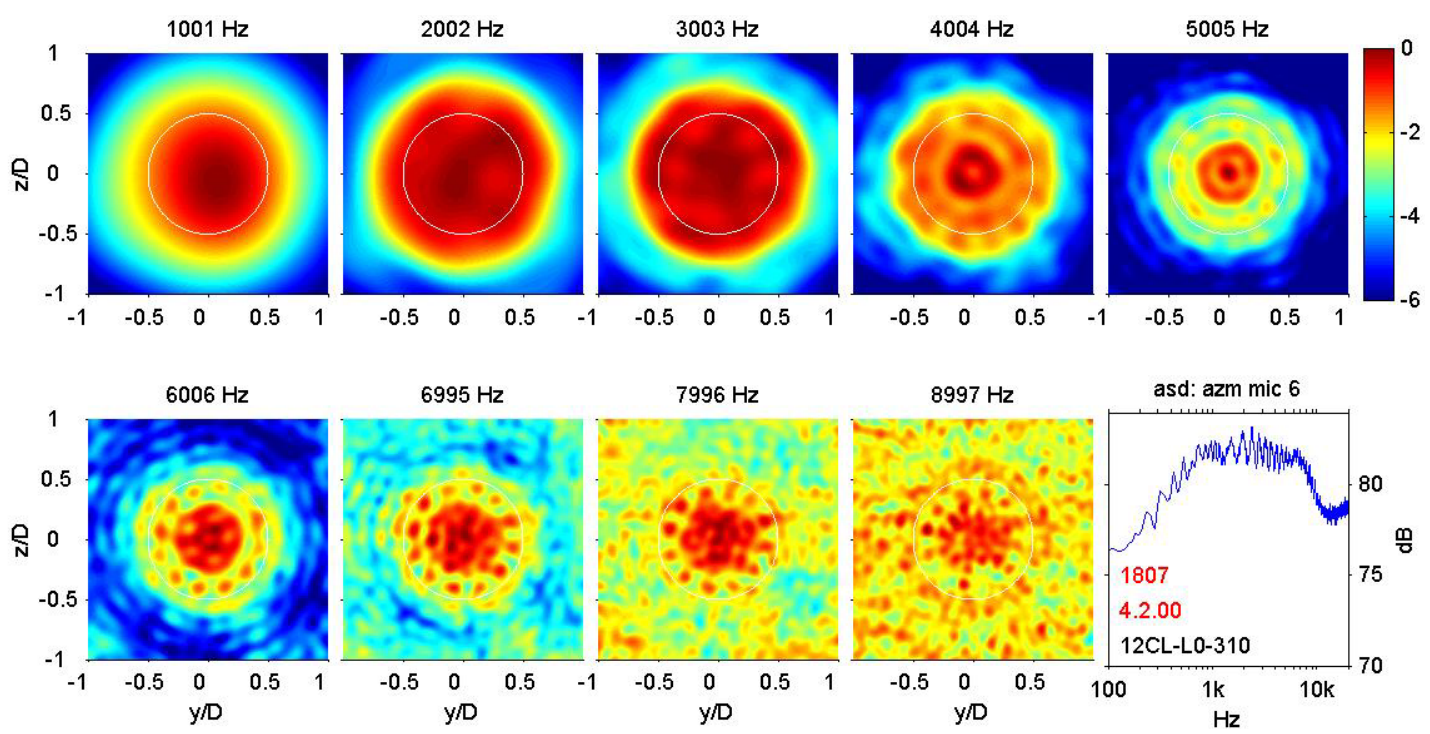

Figure 5.2.- Normalized beamforming levels from azimuthal array for 12CL mixer with L0 nozzle at set point 310 and $\mathrm{x}=0$ along with narrowband spectrum of microphone 6 (Escort number 1807). 

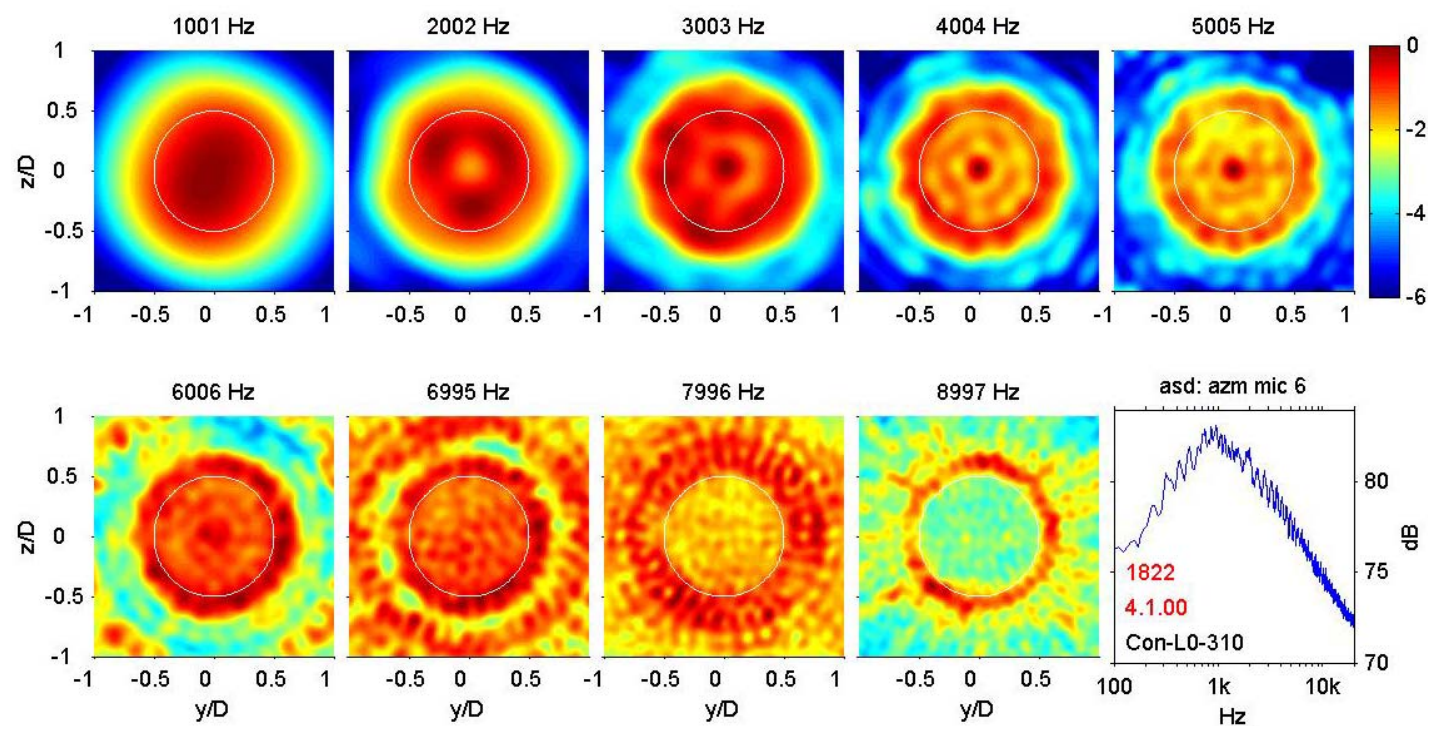

Figure 5.3.- Normalized beamforming levels from azimuthal array for Confluent splitter with L0 nozzle at set point 310 and $\mathrm{x}=0$ along with narrowband spectrum of microphone 6 (Escort number 1822).
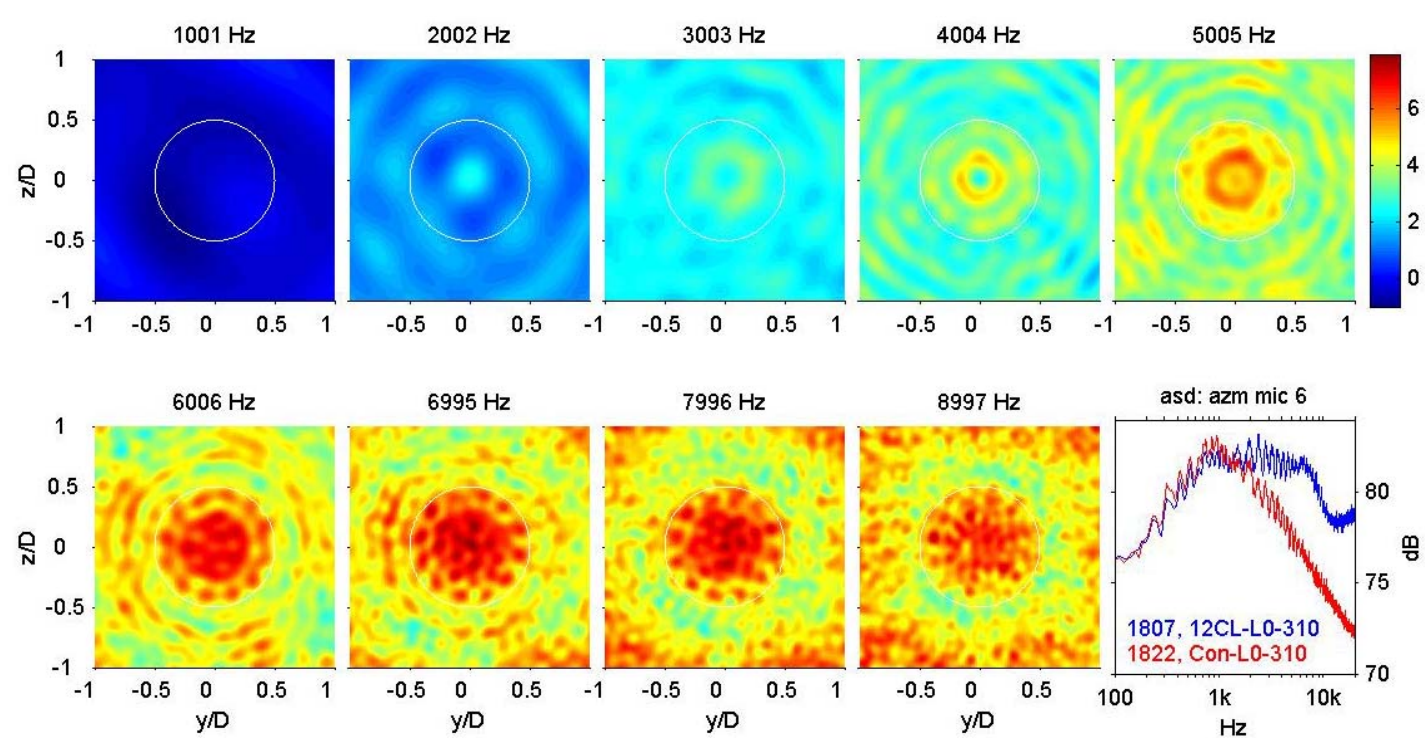

Figure 5.4.- Ratio of the beamforming level of 12CL mixer to Confluent splitter from azimuthal array for L0 nozzle at set point 310 and $\mathrm{x}=0$ along with narrowband spectra of microphone 6 (lower right). Escort numbers: 1807 and 1822. 

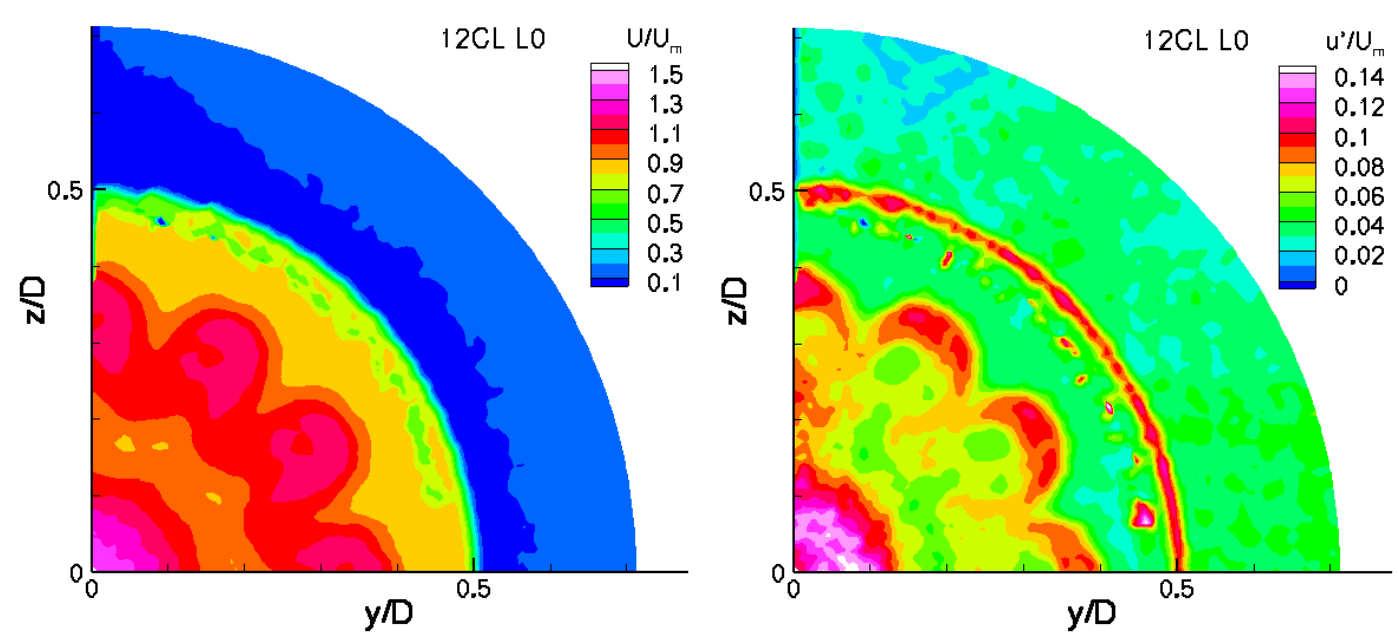

Figure 5.5.- Streamwise mean and rms turbulence velocities for 12CL mixer with L0 nozzle at 312 condition and $\mathrm{x} / \mathrm{D}=0.2$ from the Particle Image Velocimetry (PIV) tests by Bridges and Wernet (2004).
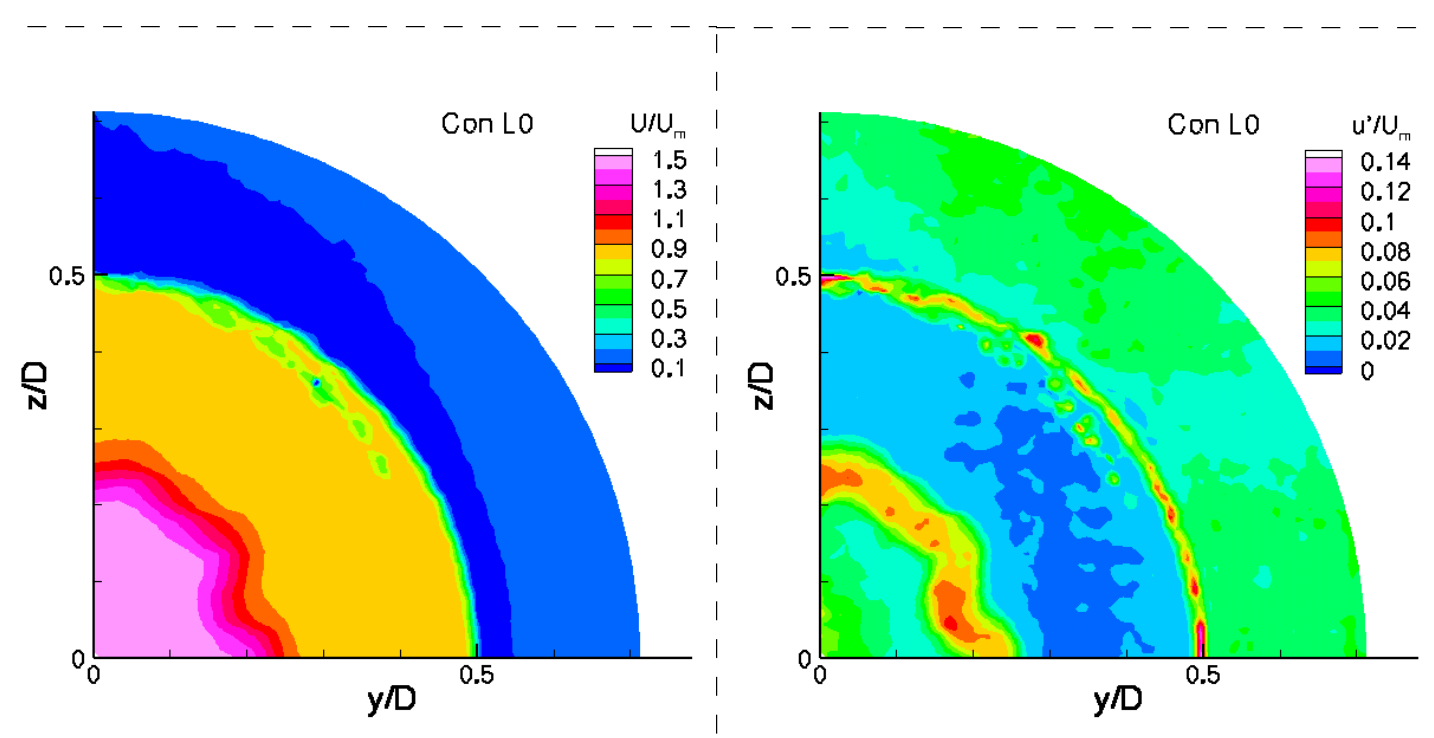

Figure 5.6.- Streamwise mean and rms turbulence velocities for Confluent splitter with L0 nozzle at 312 condition and $\mathrm{x} / \mathrm{D}=0.2$ from the Particle Image Velocimetry (PIV) tests by Bridges and Wernet (2004). 

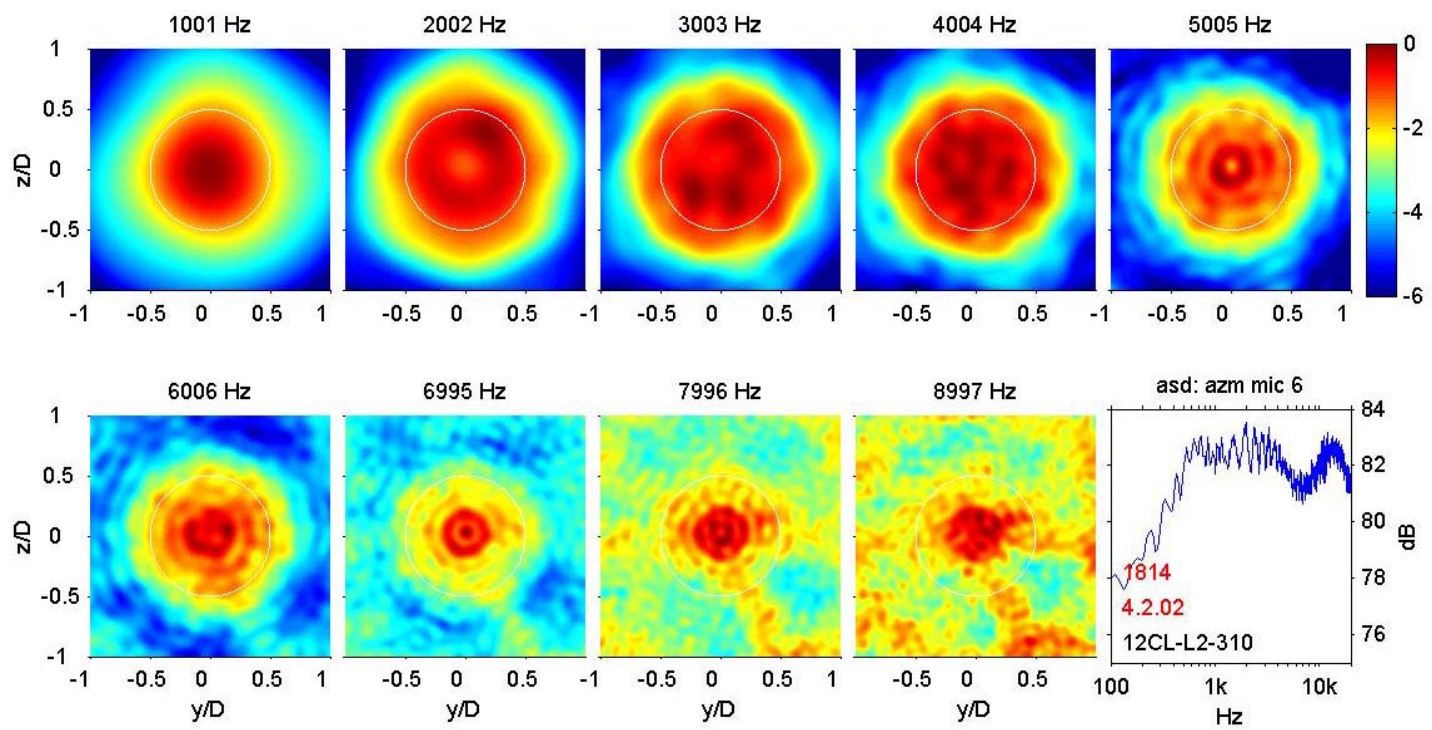

Figure 5.7.- Normalized beamforming levels from azimuthal array for 12CL mixer with L2 nozzle at set point 310 and $\mathrm{x} / \mathrm{D}=0.75$ along with narrowband spectrum of microphone 6 (Escort number 1814).
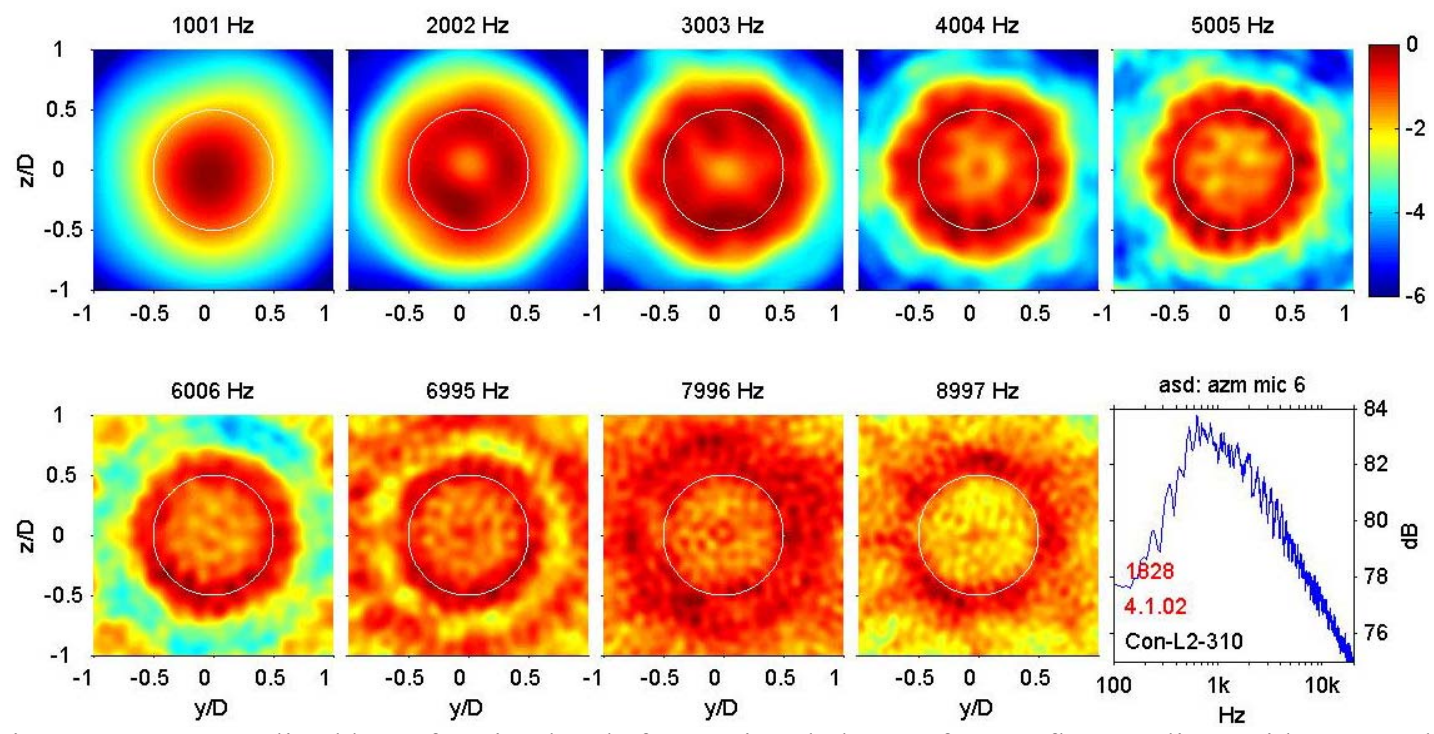

Figure 5.8.- Normalized beamforming levels from azimuthal array for Confluent splitter with L2 nozzle at set point 310 and $\mathrm{x} / \mathrm{D}=0.75$ along with narrowband spectrum of microphone 6 (Escort number 1828). 

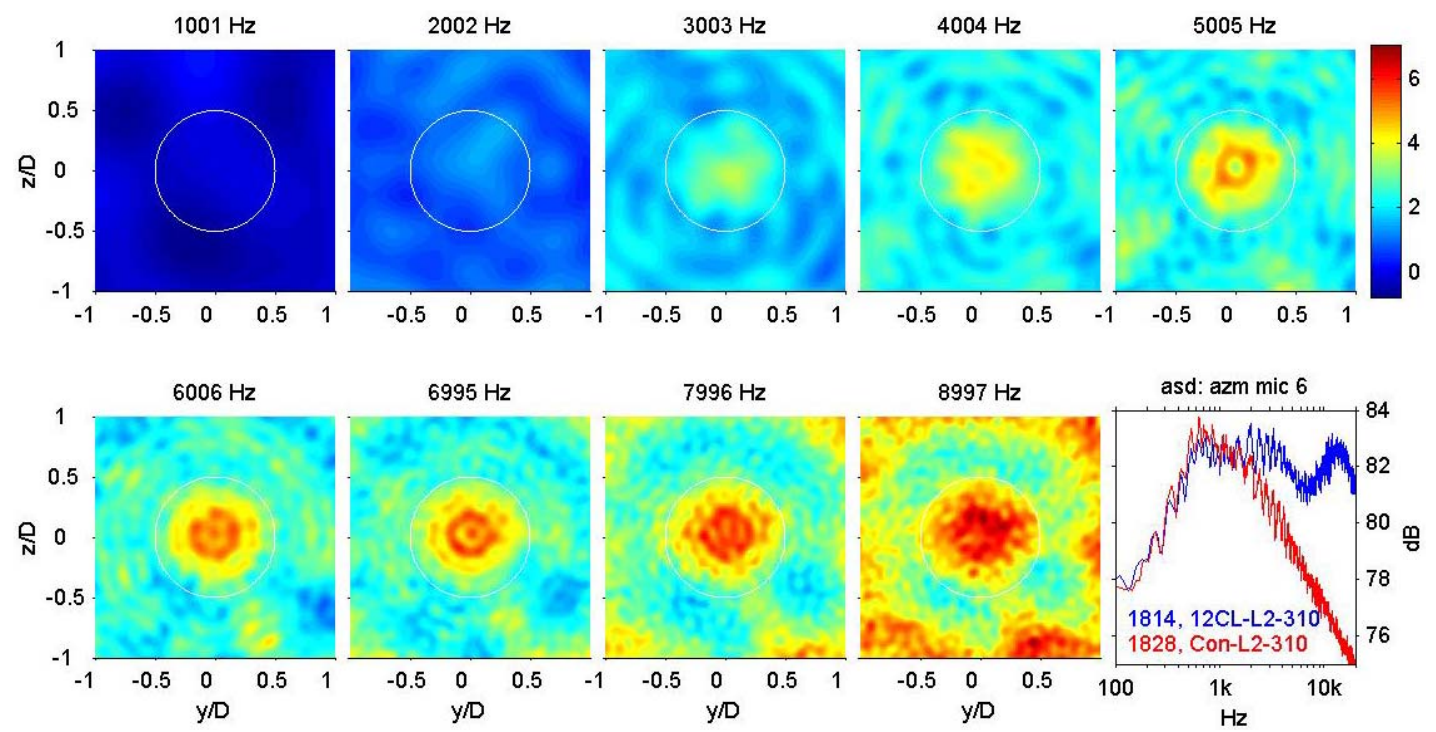

asd: azm mic 6

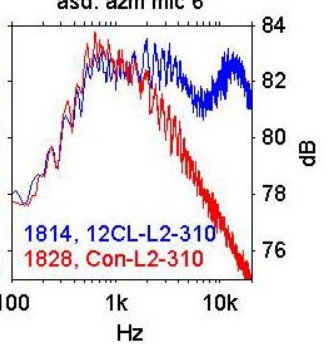

Figure 5.9.- Ratio of the beamforming level of $12 \mathrm{CL}$ mixer to Confluent splitter from azimuthal array for $\mathrm{L} 2$ nozzle at set point 310 and $\mathrm{x} / \mathrm{D}=0.75$ along with narrowband spectra of microphone 6 (lower right). Escort numbers: 1814 and 1828. 

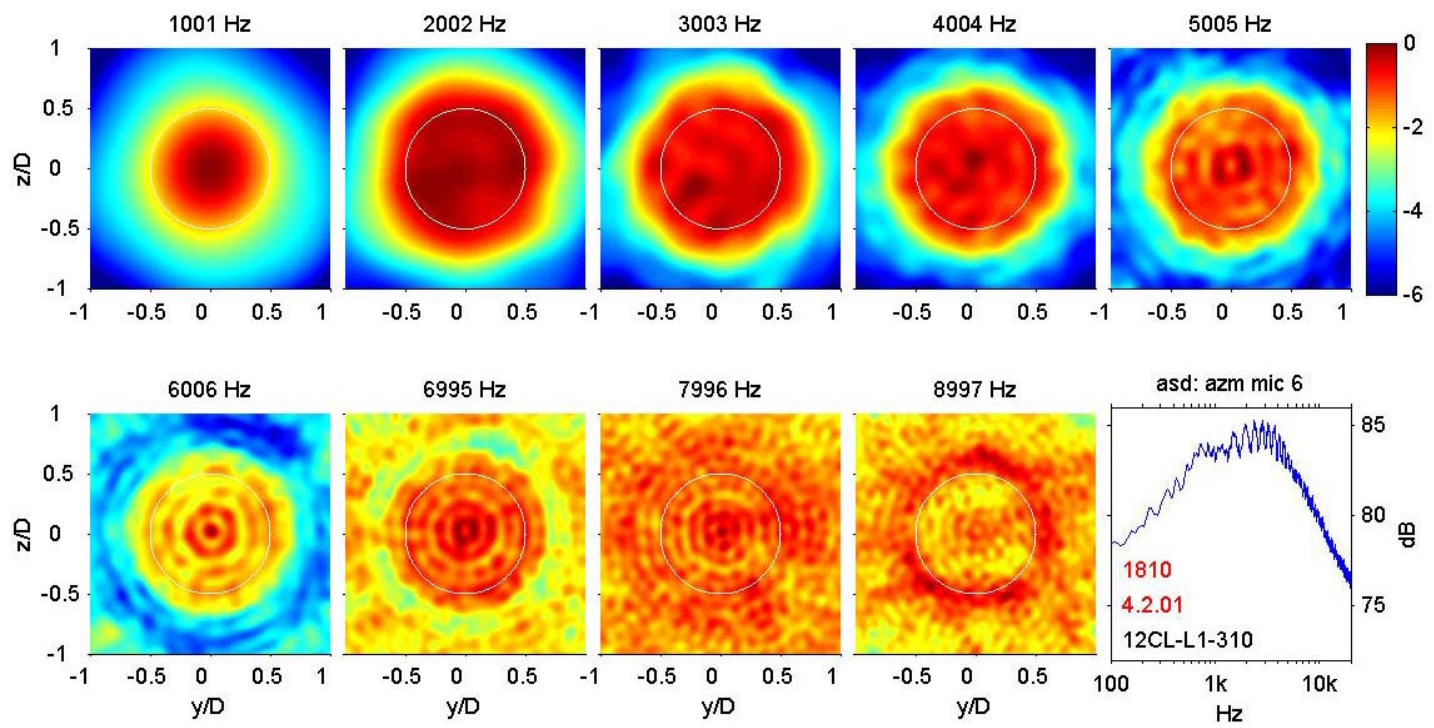

Figure 5.10.- Normalized beamforming levels from azimuthal array for 12CL mixer with L1 nozzle at set point 310 and $x / D=0.37$ along with narrowband spectrum of microphone 6 (Escort number 1810).
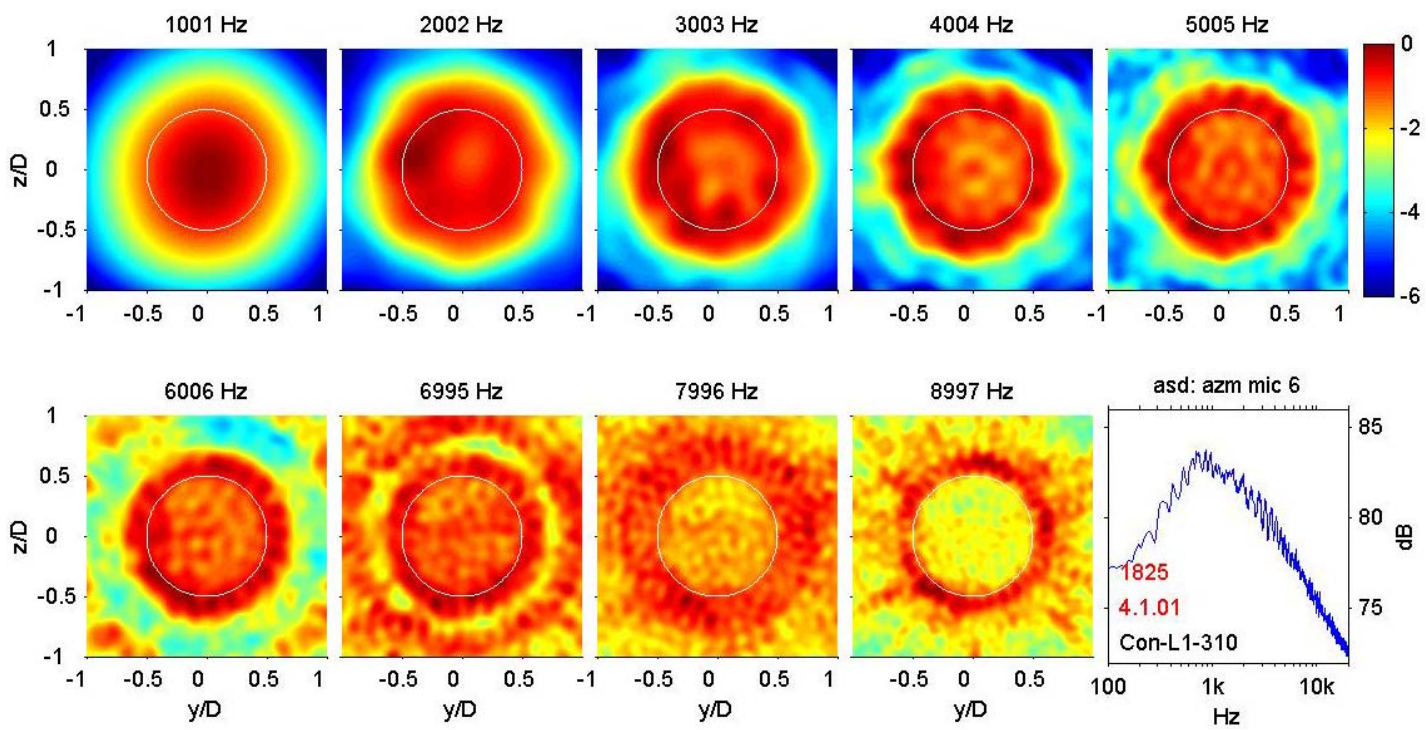

Figure 5.11.- Normalized beamforming levels from azimuthal array for Confluent splitter with L1 nozzle at set point 310 and $\mathrm{x} / \mathrm{D}=0.37$ along with narrowband spectrum of microphone 6

(Escort number 1825). 

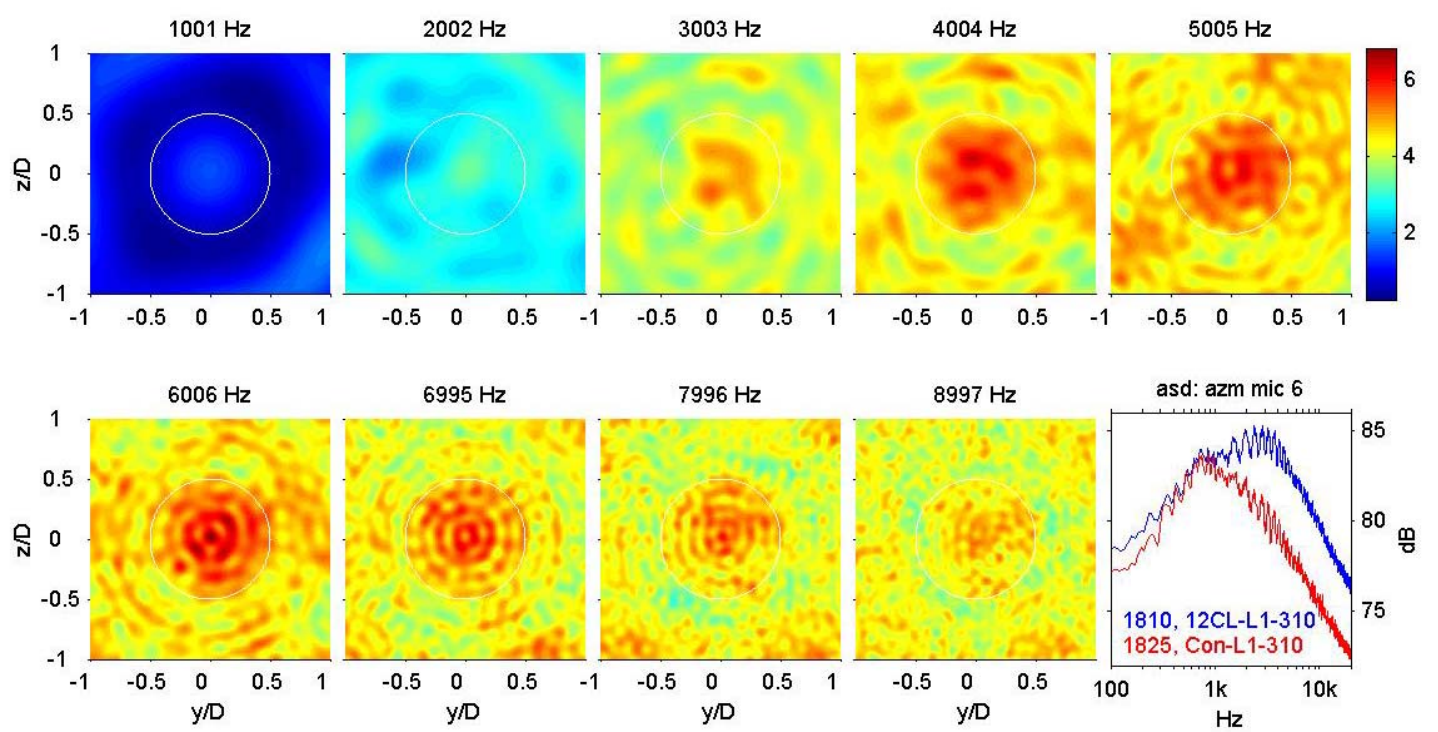

Figure 5.12.- Ratio of the beamforming level of $12 \mathrm{CL}$ mixer to Confluent splitter from azimuthal array for $\mathrm{L} 1$ nozzle at set point 310 and $\mathrm{x} / \mathrm{D}=0.37$ along with narrowband spectra of microphone 6 (lower right). Escort numbers: 1810 and 1825. 

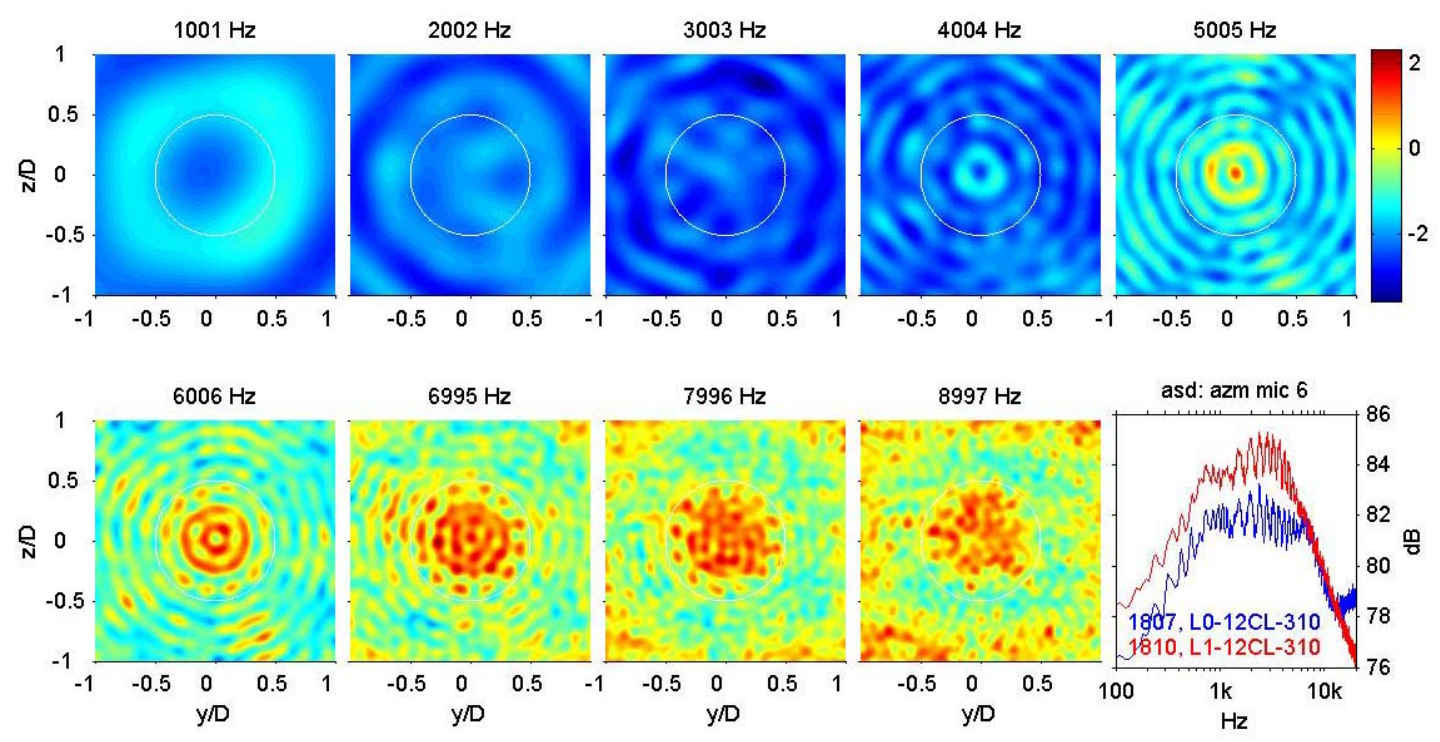

Figure 5.13.- Ratio of the beamforming level of L0 nozzle to L1 nozzle for 12CL mixer from azimuthal array at set point 310 along with narrowband spectra of microphone 6 (lower right). $\mathrm{x} / \mathrm{D}=0$ and 0.37 for L0 and L1 nozzle, respectively. Escort numbers: 1807 and 1810.
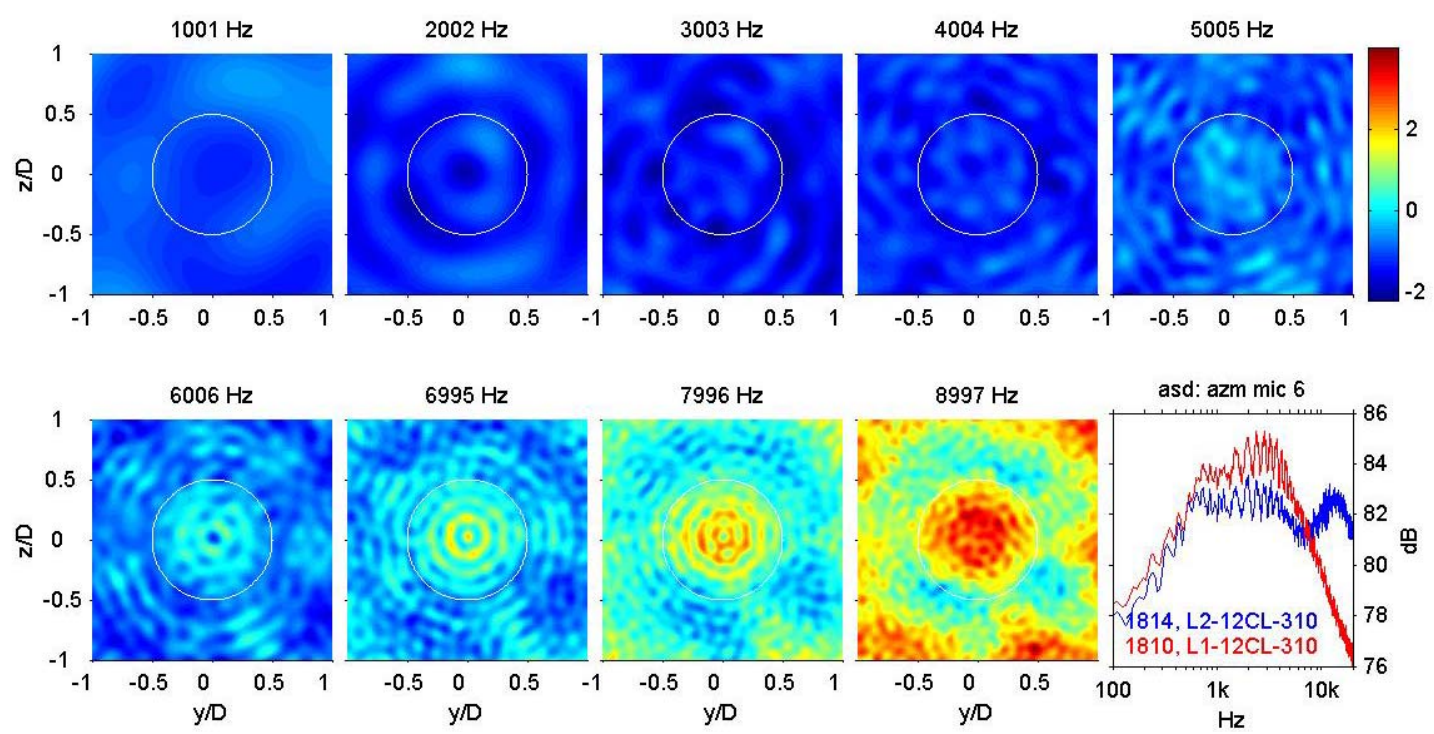

Figure 5.14.- Ratio of the beamforming level of L2 nozzle to L1 nozzle for 12CL mixer from azimuthal array at set point 310 along with narrowband spectra of microphone 6 (lower right). $\mathrm{x} / \mathrm{D}=0.75$ and 0.37 for L2 and L1 nozzle, respectively. Escort numbers: 1814 and 1810. 

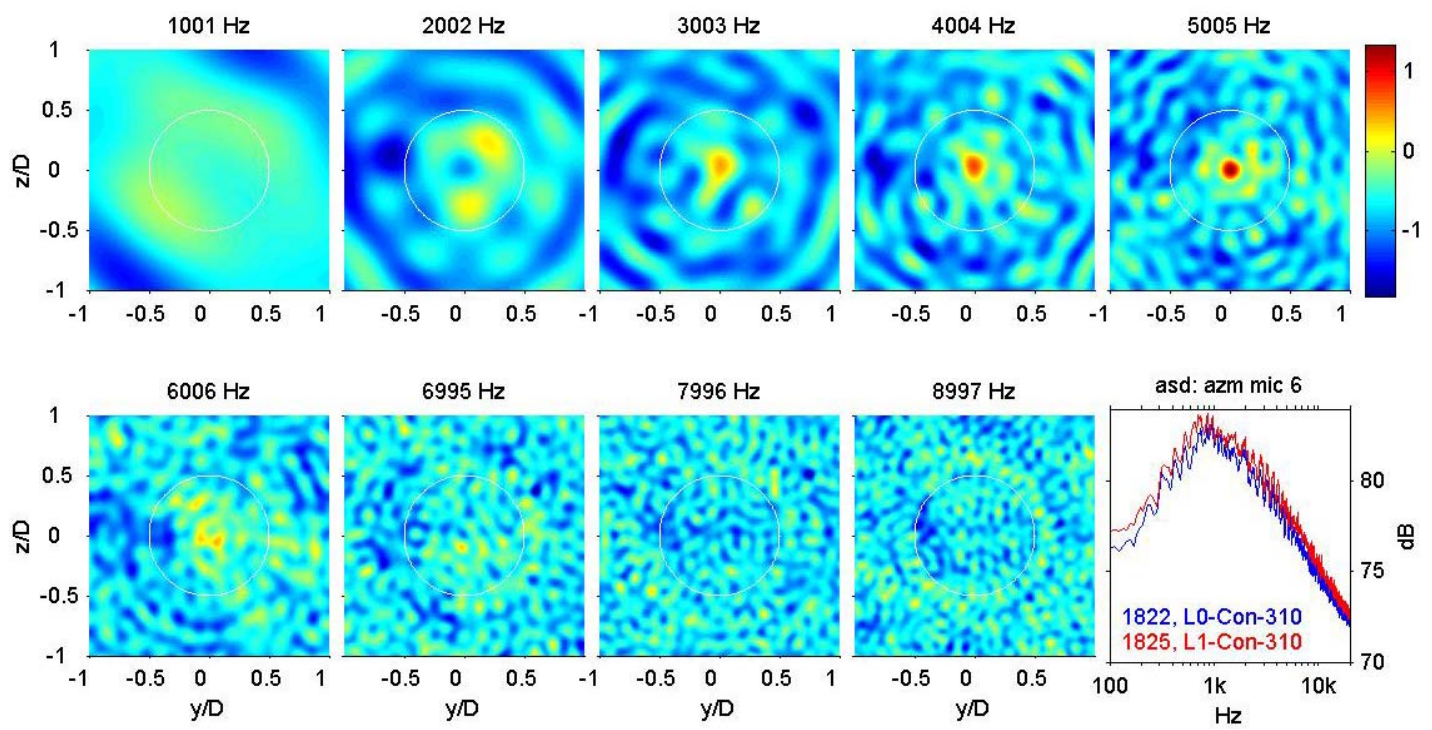

Figure 5.15.--Ratio of the beamforming level of L0 nozzle to L1 nozzle for Confluent splitter from azimuthal array at set point 310 along with narrowband spectra of microphone 6 (lower right). $\mathrm{x} / \mathrm{D}=0$ and 0.37 for L0 and L1 nozzle, respectively. Escort numbers: 1822 and 1825.
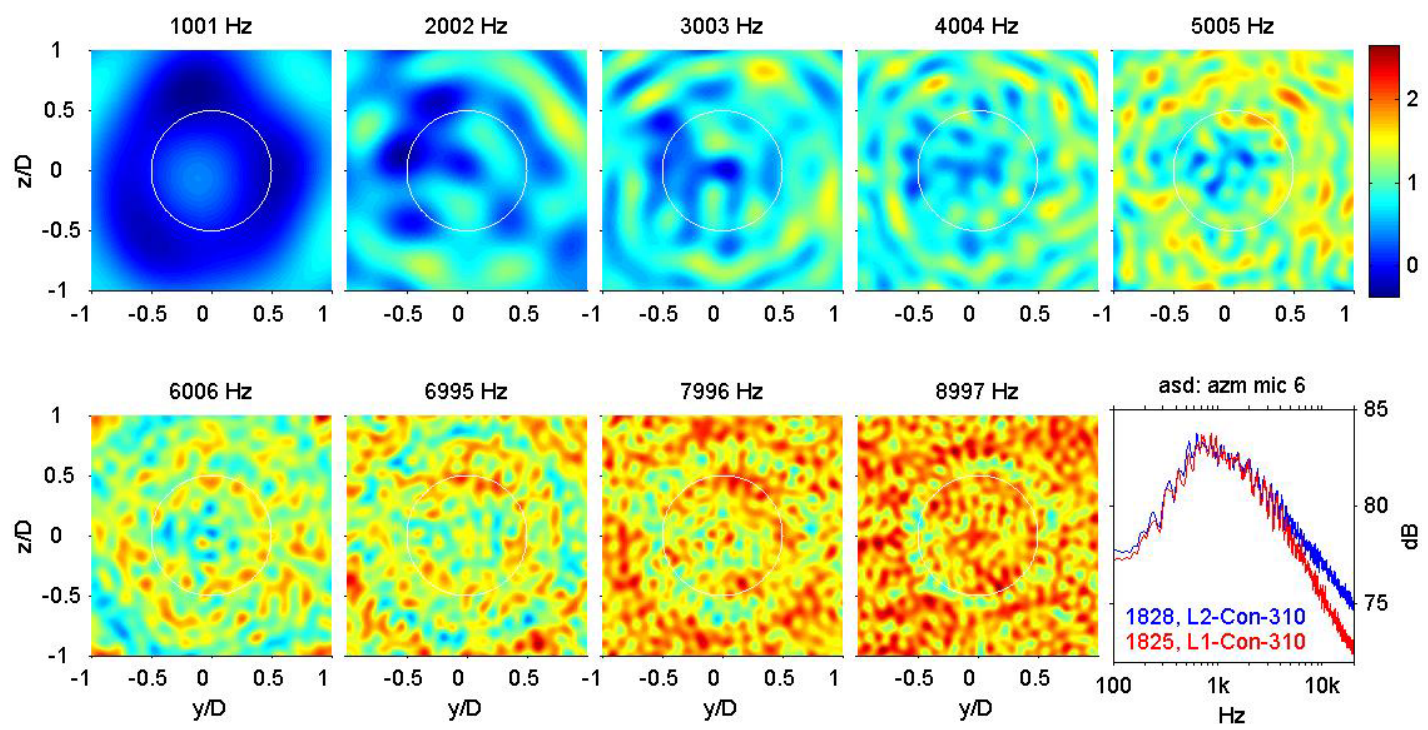

Figure 5.16.-Ratio of the beamforming level of L2 nozzle to L1 nozzle for Confluent splitter from azimuthal array at set point 310 along with narrowband spectra of microphone 6 (lower right). $\mathrm{x} / \mathrm{D}=0$ and 0.75 for L2 and L1 nozzle, respectively. Escort numbers: 1828 and 1825. 

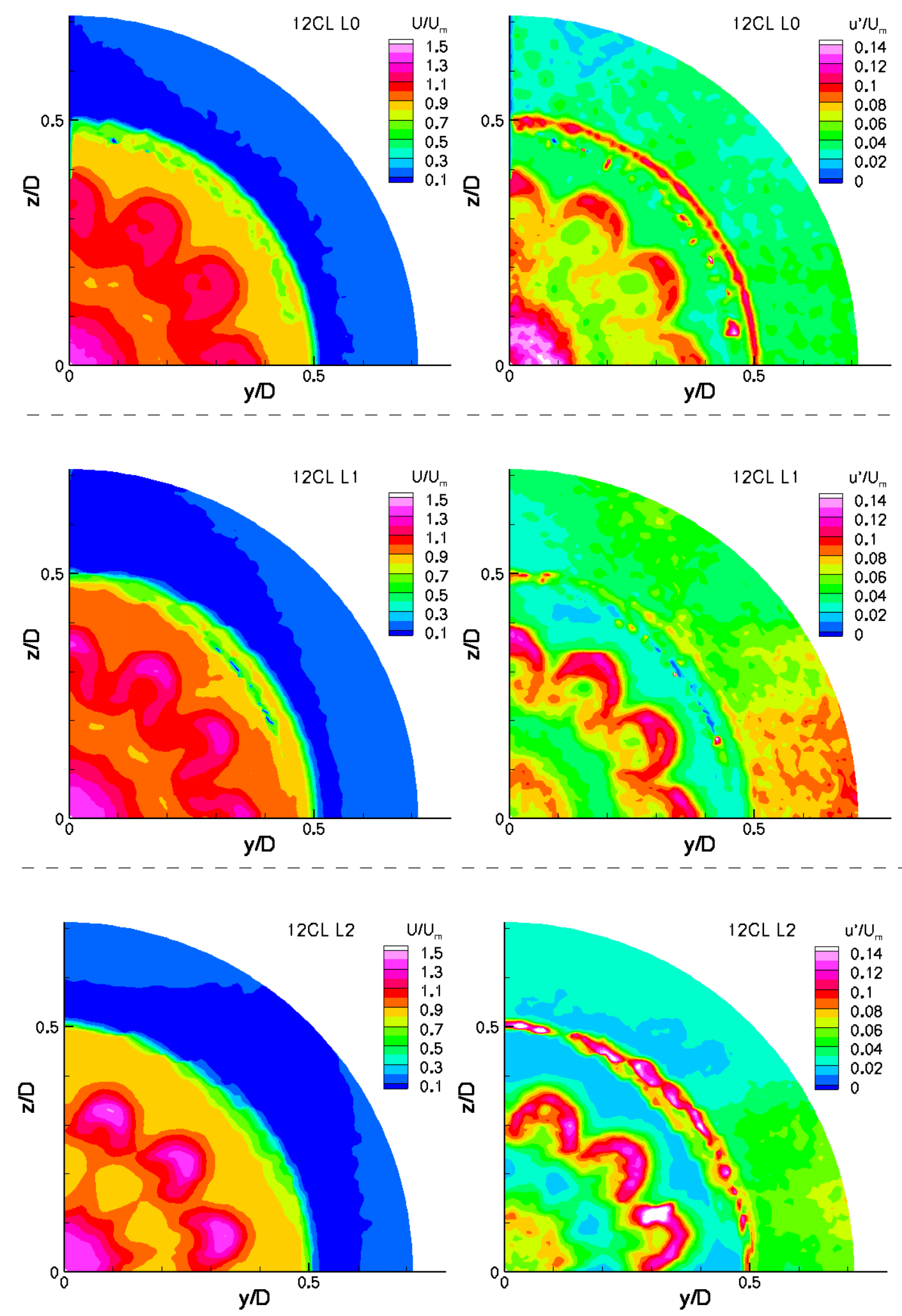

Figure 5.17.- Streamwise mean velocity and rms turbulence velocity for the 12CL mixer with L0, L1, and $\mathrm{L} 2$ nozzles at 312 condition and $\mathrm{x} / \mathrm{D}=0.2$ from the Particle Image Velocimetry (PIV) tests by Bridges and Wernet (2004). 


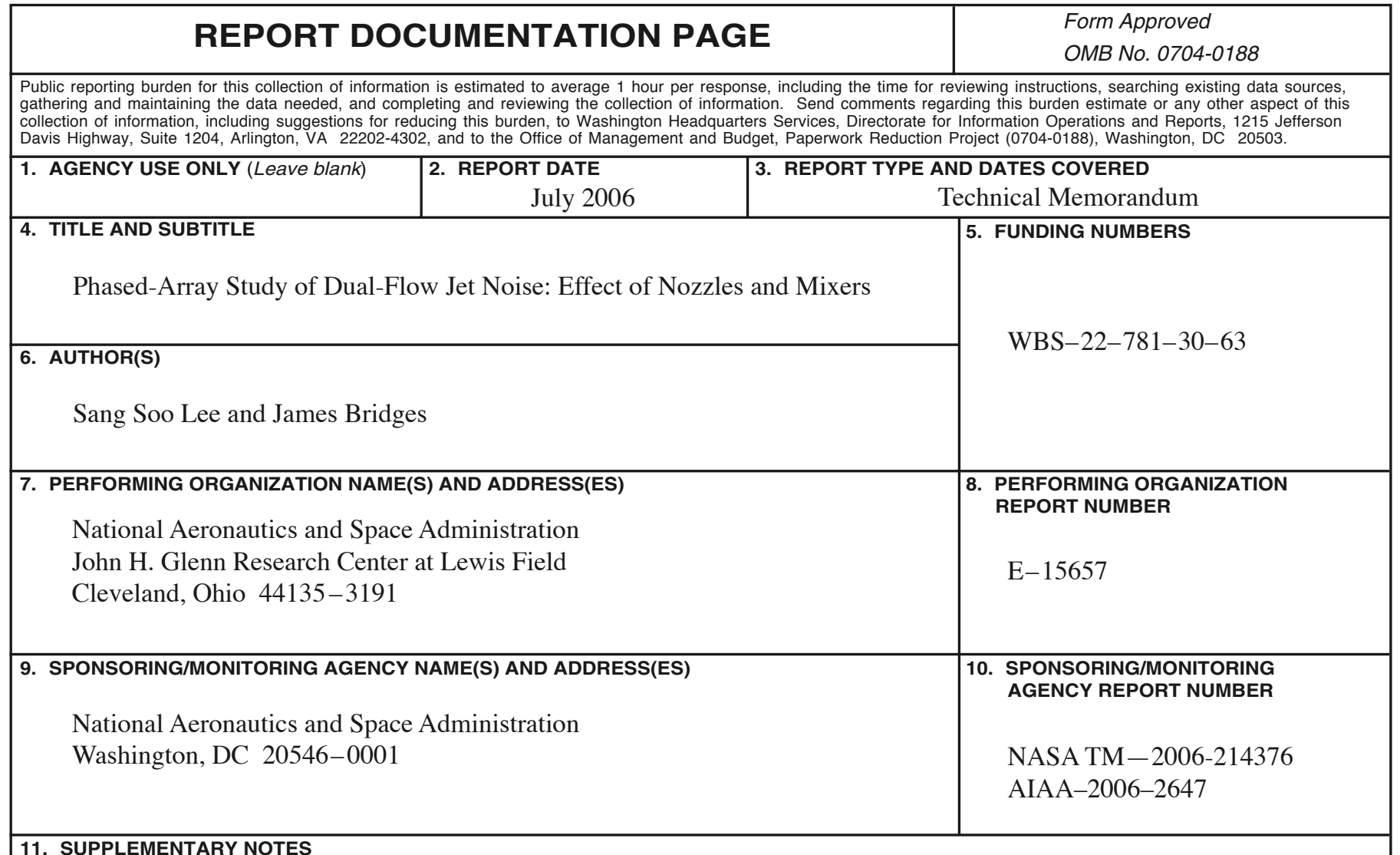

\section{SUPPLEMENTARY NOTES}

Prepared for the 12th Aeroacoustics Conference cosponsored by the American Institute of Aeronautics and Astronautics and Confederation of European Aerospace Societies, Cambridge, Massachusetts, May 8-10, 2006. Sang Soo Lee, QSS Group, Inc., 21000 Brookpark Road, Cleveland, Ohio 44135; James Bridges, NASA Glenn Research Center. Responsible person, Sang Soo Lee, organization code RTA, 216-977-1378.

12a. DISTRIBUTION/AVAILABILITY STATEMENT

12b. DISTRIBUTION CODE

Unclassified - Unlimited

Subject Categories: 07 and 34

Available electronically at http://gltrs.grc.nasa.gov

This publication is available from the NASA Center for AeroSpace Information, 301-621-0390.

13. ABSTRACT (Maximum 200 words)

A 16-microphone linear phased-array installed parallel to the jet axis and a 32-microphone azimuthal phased-array installed in the nozzle exit plane have been applied to identify the noise source distributions of nozzle exhaust systems with various internal mixers (lobed and axisymmetric) and nozzles (three different lengths). Measurements of velocity were also obtained using cross-stream stereo particle image velocimetry (PIV). Among the three nozzle lengths tested, the medium length nozzle was the quietest for all mixers at high frequency on the highest speed flow condition. Large differences in source strength distributions between nozzles and mixers occurred at or near the nozzle exit for this flow condition. The beamforming analyses from the azimuthal array for the 12-lobed mixer on the highest flow condition showed that the core flow and the lobe area were strong noise sources for the long and short nozzles. The 12 noisy spots associated with the lobe locations of the 12-lobed mixer with the long nozzle were very well detected for the frequencies $5 \mathrm{KHz}$ and higher. Meanwhile, maps of the source strength of the axisymmetric splitter show that the outer shear layer was the most important noise source at most flow conditions. In general, there was a good correlation between the high turbulence regions from the PIV tests and the high noise source regions from the phased-array measurements.

\begin{tabular}{|c|c|c|}
\hline \multicolumn{3}{|l|}{$\begin{array}{l}\text { 14. SUBJECT TERMS } \\
\text { Jets; Noise reduction; Aer }\end{array}$} \\
\hline $\begin{array}{l}\text { 17. SECURITY CLASSIFICATION } \\
\text { OF REPORT }\end{array}$ & $\begin{array}{l}\text { 18. SECURITY CLASSIFICATION } \\
\text { OF THIS PAGE }\end{array}$ & $\begin{array}{l}\text { 19. SECURITY CLASSIFICATION } \\
\text { OF ABSTRACT }\end{array}$ \\
\hline Unclassified & Unclassified & Unclassified \\
\hline
\end{tabular}

15. NUMBER OF PAGES

40

16. PRICE CODE

20. LIMITATION OF ABSTRACT

Standard Form 298 (Rev. 2-89)

Prescribed by ANSI Std. Z39-18 298-102 

